\title{
Existence of Nonradial Solutions for Hénon Type Biharmonic Equation Involving Critical Sobolev Exponents
}

\author{
Yajing Zhang, Ningning Wang, Yinmei Lü, and Jianghao Hao \\ School of Mathematical Sciences, Shanxi University, Taiyuan, Shanxi 030006, China \\ Correspondence should be addressed to Jianghao Hao; hjhao@sxu.edu.cn
}

Received 13 June 2014; Accepted 19 August 2014; Published 14 October 2014

Academic Editor: Antonio Suárez

Copyright (C) 2014 Yajing Zhang et al. This is an open access article distributed under the Creative Commons Attribution License, which permits unrestricted use, distribution, and reproduction in any medium, provided the original work is properly cited.

We prove the existence of nonradial solutions under some conditions for a semilinear biharmonic Dirichlet problem involving critical Sobolev exponents.

\section{Introduction}

In this paper we consider the following Hénon type biharmonic problem:

$$
\begin{gathered}
\Delta^{2} u=|x|^{\alpha}|u|^{2^{*}-2} u \quad \text { in } \Omega, \\
u=\frac{\partial u}{\partial \mathbf{n}}=0 \quad \text { on } \partial \Omega,
\end{gathered}
$$

where $\alpha \geq 0,2^{*}=2 N /(N-4), \Omega$ is the unit ball of $\mathbb{R}^{N}, N \geq 5$, and $\mathbf{n}$ denotes the unit outward normal at the boundary $\partial \Omega$.

We consider first the case where $\alpha=0$, namely, the equation

$$
\begin{aligned}
& \Delta^{2} u=|u|^{2^{*}-2} u \quad \text { in } \Omega, \\
& u=\frac{\partial u}{\partial \mathbf{n}}=0 \quad \text { on } \partial \Omega .
\end{aligned}
$$

It is well known that (2) admits no nontrivial radial solution (see [1], Theorem 3.11, or [2], Theorem 4). The nonexistence of any nontrivial solution to (2) seems to be still unknown; only more restricted results are available. In order to obtain existence results for (2), one should either add subcritical perturbations or modify the topology or the geometry of the domain. For subcritical perturbations, we refer to $[2,3]$ and references therein. Domains with nontrivial topology are studied in $[2,4]$. They demonstrated how domains with topology often carry solutions that cannot be present otherwise. The corresponding second order elliptic problem has been investigated by Bahri and Coron in [5].

Berchio et al. [6], among other things, considered the minimization problem

$$
\inf _{u \in H_{0, \mathrm{rad}}^{2}(\Omega) \backslash\{0\}} \frac{\int_{\Omega}|\Delta u|^{2} d x}{\left(\int_{\Omega}|x|^{\alpha}|u|^{2^{*}} d x\right)^{2 / 2^{*}}},
$$

where $H_{0 \text {,rad }}^{2}(\Omega)$ denotes the subspace of radial functions in $H_{0}^{2}(\Omega)$. Actually, they treated general polyharmonic problem. They proved the infimum in (3) is attained. The minimizers of (3), after rescaling, are a solution of (1). It is natural to ask whether (1) has a nontrivial nonradial solution. We will answer this problem partially here.

Our main result is as follows.

Theorem 1. Let $N \geq 8$ and let $\Omega$ be the unit ball in $\mathbb{R}^{N}$. Then, for every $\alpha>0$ large enough, problem (1) admits at least one nonradial solution.

The corresponding second order elliptic problem, namely, the Hénon equation,

$$
\begin{gathered}
-\Delta u=|x|^{\alpha}|u|^{p-1}, \quad u>0 \text { in } \Omega, \\
u=0 \quad \text { on } \partial \Omega,
\end{gathered}
$$

has been studied by many authors, where $p>1$. Ni [7], among other things, proved the existence of radial positive 
solutions of (4) for all $p \in(1,(N+2+2 \alpha) /(N-2))$. For the case $p \in(1,(N+2) /(N-2))$, there are many works concerning the limiting behavior of the ground state solutions of (4); see, for example, [8-11] and the references therein. In particular, Smets et al. [11] proved that, for every $p \in(1,(N+2) /(N-2))$, no minimizer of

$$
\frac{\int_{\Omega}|\nabla u|^{2} d x}{\left(\int_{\Omega}|x|^{\alpha}|u|^{p} d x\right)^{2 / p}}
$$

is radial provided $\alpha$ is large enough. Serra [12] studied the case $p=(N+2) /(N-2)$ and proved the existence of nonradial positive solutions of (4) for $\alpha$ large. Theorem 1 can be regarded as an extension of Serra's result to biharmonic problem.

In order to outline the proof of Theorem 1, we introduce some notations. We write $\mathbb{R}^{N}=\mathbb{R}^{2} \times \mathbb{R}^{N-2} \simeq \mathbb{C} \times \mathbb{R}^{N-2}$ and $x=(z, y)$. For a given integer $k$, let $G_{k}$ be the group $\mathbb{Z}_{k} \times$ $\mathbf{O}(N-2)$. We consider the action of $G_{k}$ on $H_{0}^{2}(\Omega)$ given by

$$
g(u)(x)=g(u)(z, y)=u\left(e^{j(2 \pi i / k)} z, R y\right),
$$

where $j \in\{0,1, \ldots, k-1\}$ and $R \in \mathbf{O}(N-2)$.

Define

$$
\begin{aligned}
H_{k}=\{u & \in H_{0}^{2}(\Omega): u\left(e^{2 \pi i / k} z, R y\right) \\
= & u(z, y), \forall R \in \mathbf{O}(N-2)\} .
\end{aligned}
$$

It is easy to see that functions in $H_{k}$ are radial in $y$. Since both the numerator and the denominator of the functional $Q_{\alpha}$ are invariant under the action of $G_{k}$, the functional $Q_{\alpha}$ is invariant. So the critical points of $Q_{\alpha}$ restricted to $H_{k}$ are critical points of $Q_{\alpha}$. After scaling, these correspond to weak solution of (1), which are in fact classical solutions by standard elliptic theory (see $[13,14])$.

Set

$$
\begin{aligned}
Q_{\alpha}(u) & =\frac{\int_{\Omega}|\Delta u|^{2} d x}{\left(\int_{\Omega}|x|^{\alpha}|u|^{2^{*}} d x\right)^{2 / 2^{*}}}, \\
\Sigma_{k} & =\inf _{u \in H_{k} \backslash\{0\}} Q_{\alpha}(u) .
\end{aligned}
$$

Notice that since $|x| \leq 1$ in $\Omega$, we have $\Sigma_{k} \geq S$, the best Sobolev constant for the embedding $H_{0}^{2} \hookrightarrow L^{2^{*}}$; that is,

$$
S=\inf _{u \in H_{0}^{2}(\Omega) \backslash\{0\}} \frac{\int_{\Omega}|\Delta u|^{2} d x}{\left(\int_{\Omega}|u|^{2^{*}} d x\right)^{2 / 2^{*}}} .
$$

We now briefly outline the proof of Theorem 1. Firstly, we show that, for every $\alpha>0$, there exists $k_{\alpha}>0$ such that $\Sigma_{k}<$ $k^{4 / N} S$ for every integer $k \geq k_{\alpha}$. Next we prove that if $\Sigma_{k}<$ $k^{4 / N} S$, then $\Sigma_{k}$ is achieved. Finally, we obtain a bound from below for $\inf _{u \in H_{0, \text { rad }}^{2}(\Omega) \backslash\{0\}} Q_{\alpha}(u)$ and then prove

$$
\Sigma_{k}<\inf _{u \in H_{0, \text { rad }}^{2}(\Omega) \backslash\{0\}} Q_{\alpha}(u)
$$

for all $\alpha$ large enough, where $H_{0, \text { rad }}^{2}(\Omega)$ denotes the space of radial functions in $H_{0}^{2}(\Omega)$. Therefore, our solution cannot be radial.

The paper is organized as follows. In Section 2, we establish some estimates we will need and investigate the compactness properties of Palais-Smale sequences for $Q_{\alpha}$. In Section 3, we prove Theorem 1. Throughout this paper, the constant $C$ will denote various generic constants.

\section{Asymptotic Estimates and Analysis of Palais-Smale Sequences}

In this section, we first establish the estimate to prove that $\Sigma<k^{4 / N} S$ for suitable values of $k$ and $\alpha$.

In what follows, when we need to, we may define the trivial extension of functions $u$ in $H_{0}^{2}(\Omega)$ by zero; namely, $u(x)=0$ for $x \in \mathbb{R}^{N} \backslash \Omega$. For any fixed $\lambda>0, q \in \mathbb{R}^{N}$, the rescaling $\mathscr{T}=\mathscr{T}(\lambda, q): D^{2,2}\left(\mathbb{R}^{N}\right) \rightarrow D^{2,2}\left(\mathbb{R}^{N}\right)$ is defined by

$$
\mathscr{T} v(x)=\lambda^{-(N-4) / 2} v\left(\frac{x}{\lambda}+q\right) \text {. }
$$

Notice that $\mathscr{T}^{-1}=\mathscr{T}(1 / \lambda,-\lambda q)$; that is,

$$
\mathscr{T}^{-1} v(x)=\lambda^{(N-4) / 2} v(\lambda(x-q)) .
$$

We choose $r \in(0,1)$ and for every $k \in \mathbb{N}$ we define $k$ points $x^{(j)}$ in $\mathbb{R}^{N} \cong \mathbb{C} \times \mathbb{R}^{N-2}$ as

$$
x^{(j)}=\left((1-r) e^{j(2 \pi i / k)}, 0\right), \quad j=0,1, \ldots, k-1 .
$$

Notice that the points $x^{(j)}$ are all in $\Omega$. Define

$$
\begin{aligned}
U_{\lambda, x^{(j)}}(x) & =\mathscr{T}\left(\lambda, x^{(j)}\right)^{-1} U(x) \\
& =\frac{C_{N} \lambda^{(N-4) / 2}}{\left(1+\lambda^{2}\left|x-x^{(j)}\right|^{2}\right)^{(N-4) / 2}},
\end{aligned}
$$

where $C_{N}=[(N-4)(N-2) N(N+2)]^{(N-4) / 8}$. We recall that $U_{\lambda, x^{(j)}}$ are the unique positive solutions, radial about $x^{(j)}$, of the equation $\Delta^{2} U=U^{2^{*}-1}$ in $D^{2,2}\left(\mathbb{R}^{N}\right)$, and $\int_{\mathbb{R}^{N}}\left|\Delta U_{\lambda, x^{(j)}}\right|^{2} d x=\int_{\mathbb{R}^{N}} U_{\lambda, x^{(j)}}^{2^{*}} d x=S^{N / 4}$ for all $\lambda$ and $x^{(j)}$. To fix ideas, we anticipate that we will let $r \rightarrow 0, n \rightarrow \infty$, and $\lambda \rightarrow \infty$, with appropriate relations between $r, n$, and $\lambda$. The functions $U_{\lambda, x^{(j)}}$ are not in $H_{0}^{2}(\Omega)$, so we will use instead their projections $P U_{\lambda, x^{(j)}}$ on $H_{0}^{2}(\Omega)$ defined by

$$
\begin{aligned}
& \Delta^{2} P U_{\lambda, x^{(j)}}=\Delta^{2} U_{\lambda, x^{(j)}} \text { in } \Omega, \\
& P U_{\lambda, x^{(j)}}=\frac{\partial P U_{\lambda, x^{(j)}}}{\partial \mathbf{n}}=0 \quad \text { on } \partial \Omega .
\end{aligned}
$$

By Lemma 2.27 in [15], we have $P U_{\lambda, x^{(j)}}(x)>0$ in $\Omega$. If we set $\varphi_{\lambda, x^{(j)}}=U_{\lambda, x^{(j)}}-P U_{\lambda, x^{(j)}}$, then

$$
\begin{gathered}
\Delta^{2} \varphi_{\lambda, x^{(j)}}=0 \quad \text { in } \Omega, \\
\varphi_{\lambda, x^{(j)}}=U_{\lambda, x^{(j)}} \quad \text { on } \partial \Omega, \\
\frac{\partial \varphi_{\lambda, x^{(j)}}}{\partial \mathbf{n}}=\frac{\partial U_{\lambda, x^{(j)}}}{\partial \mathbf{n}} \quad \text { on } \partial \Omega .
\end{gathered}
$$


To avoid heavy notation from now on, we will write simply $U_{j}$ for $U_{\lambda, x^{(j)}}, P U_{j}$ for $P U_{\lambda, x^{(j)}}$, and $\varphi_{j}$ for $\varphi_{\lambda, x^{(j)}}$.

We set

$$
\begin{gathered}
d_{j l}=x^{(l)}-x^{(j)}, \\
d=\frac{1}{2} \min _{j \neq l}\left|d_{j l}\right|=\frac{1}{2}\left|x^{(1)}-x^{(0)}\right|,
\end{gathered}
$$

and we assume that $2 d \leq r$ and $\lambda d \geq 1$ for all $\lambda$ under consideration. Note also that due to the definition of $x^{(j)}$, we have

$$
d=2(1-r) \sin \frac{\pi}{k} \sim \frac{C}{k}
$$

for all $r$ small.

\section{Lemma 2.}

$$
\begin{aligned}
& \int_{\Omega} U_{j}^{2^{*}-1} U_{l} d x j=l, \\
&= \begin{cases}S^{N / 4}+O\left((\lambda r)^{-N}\right), & \\
\frac{C_{N} a_{0}}{\left(\lambda\left|d_{j l}\right|\right)^{N-4}}+O\left(\frac{1}{\left(\lambda\left|d_{j l}\right|\right)^{N-2}}\right) & j \neq l \\
+O\left(\frac{1}{(\lambda r)^{N}}\right), & j\end{cases}
\end{aligned}
$$

where $a_{0}=\int_{\mathbb{R}^{N}} U^{2^{*}-1} d x$.

Proof.

Case 1. Consider $j=l$.

Direct computations yield that

$$
\begin{aligned}
S^{N / 4} & \geq \int_{\Omega} U_{j}^{2^{*}} d x \\
& \geq \int_{B_{r}\left(x^{(j)}\right)} U_{j}^{2^{*}} d x \\
& =S^{N / 4}-\int_{\mathbb{R}^{N} \backslash B_{r}\left(x^{(j)}\right)} U_{j}^{2^{*}}, \\
\int_{\mathbb{R}^{N} \backslash B_{r}\left(x^{(j)}\right)} & C_{N}^{2^{*}} \int_{\mathbb{R}^{N} \backslash B_{\lambda r}(0)} \frac{d x}{\left(1+|x|^{2}\right)^{(N-4) / 2}} \\
& \geq C_{N}^{2^{*}} \omega_{N} \int_{\lambda r}^{+\infty} \frac{\rho^{N-1} d \rho}{\left(1+\rho^{2}\right)^{N}} \\
& \leq C(\lambda r)^{-N},
\end{aligned}
$$

where $\omega$ denotes surface area of unit sphere in $\mathbb{R}^{N}$. Combining (21), we prove the first case of Lemma 2.

Case 2. Consider $j \neq l$.

Writing

$$
\int_{\Omega} U_{j}^{2^{*}-1} U_{l} d x=\int_{\mathbb{R}^{N}} U_{j}^{2^{*}-1} U_{l} d x-\int_{\mathbb{R}^{N} \backslash \Omega} U_{j}^{2^{*}-1} U_{l} d x,
$$

with the same type of calculation as in the proof of the first case, we see that

$$
\begin{aligned}
& \int_{\mathbb{R}^{N} \backslash \Omega} U_{j}^{2^{*}-1} U_{l} d x \\
& \quad \leq\left(\int_{\mathbb{R}^{N} \backslash \Omega} U_{j}^{2^{*}}\right)^{\left(2^{*}-1\right) / 2^{*}}\left(\int_{\mathbb{R}^{N} \backslash \Omega} U_{l}^{2^{*}} d x\right)^{1 / 2^{*}} \\
& \quad \leq \frac{C}{(\lambda r)^{N}} .
\end{aligned}
$$

To estimate the integral over $\mathbb{R}^{N}$ in (22), we follow exactly the calculation in [5] (see page 279-280). It is easy to see that

$$
\begin{aligned}
& \int_{\mathbb{R}^{N}} U_{j}^{2^{*}-1} U_{l} d x \\
& =C_{N}^{2^{*}} \int_{\mathbb{R}^{N}} \frac{d x}{\left(1+|x|^{2}\right)^{(N+4) / 2}\left(1+\left|x-\lambda d_{j l}\right|\right)^{(N-4) / 2}}
\end{aligned}
$$

We have also

$$
1+\left|x-\lambda d_{j l}\right|^{2}=\left(1+\lambda^{2}\left|d_{j l}\right|^{2}\right)\left(1+\frac{|x|^{2}-2 \lambda x \cdot d_{j l}}{1+\lambda^{2}\left|d_{j l}\right|^{2}}\right) .
$$

Hence

$$
\begin{aligned}
(1+\mid x & \left.-\left.\lambda d_{j l}\right|^{2}\right)^{(4-N) / 2} \\
= & \left(1+\lambda^{2}\left|d_{j l}\right|^{2}\right)^{(4-N) / 2} \\
& \times\left[1+\frac{(N-4) \lambda x \cdot d_{j l}}{1+\lambda^{2}\left|d_{j l}\right|^{2}}+O\left(\frac{|x|^{2}}{1+\lambda^{2}\left|d_{j l}\right|^{2}}\right)\right]
\end{aligned}
$$

for $|x| \leq(1 / 4) \lambda\left|d_{j l}\right|$.

Direct calculations yield

$$
\begin{aligned}
\int_{|x| \leq(1 / 4) \lambda\left|d_{j l}\right|} \frac{d x}{\left(1+|x|^{2}\right)^{(N+4) / 2}\left(1+\left|x-\lambda d_{j l}\right|^{2}\right)^{(N-4) / 2}} \\
=\frac{1}{\left(1+\lambda^{2}\left|d_{j l}\right|^{2}\right)^{(N-4) / 2}}
\end{aligned}
$$




$$
\begin{aligned}
\times & {\left[\int_{|x| \leq(1 / 4) \lambda\left|d_{j l}\right|} \frac{d x}{\left(1+|x|^{2}\right)^{(N+4) / 2}}\right.} \\
& +\frac{1}{1+\lambda^{2}\left|d_{j l}\right|^{2}} O \\
& \left.\times\left(\int_{|x| \leq(1 / 4) \lambda\left|d_{j l}\right|} \frac{|x|^{2} d x}{\left(1+|x|^{2}\right)^{(N+4) / 2}}\right)\right] \\
\int_{|x| \leq(1 / 4) \lambda\left|d_{j l}\right|} & \frac{|x|^{2} d x}{\left(1+|x|^{2}\right)^{(N+4) / 2}}=b_{0}+O\left(\frac{1}{\lambda^{2}\left|d_{j l}\right|^{2}}\right)
\end{aligned}
$$

where $b_{0}=\int_{\mathbb{R}^{N}}|x|^{2} d x /\left(1+|x|^{2}\right)^{(N+4) / 2}$, and

$$
\int_{|x| \leq(1 / 4) \lambda\left|d_{j l}\right|} \frac{d x}{\left(1+|x|^{2}\right)^{(N+4) / 2}}=\frac{a_{0}}{C_{N}^{2^{*}-1}}+O\left(\frac{1}{\lambda^{4}\left|d_{j l}\right|^{4}}\right) .
$$

From (27)-(29), we obtain

$$
\begin{gathered}
\int_{|x| \leq(1 / 4) \lambda \mid d_{j l} l} \frac{d x}{\left(1+|x|^{2}\right)^{(N+4) / 2}\left(1+\left|x-\lambda d_{j l}\right|^{2}\right)^{(N-4) / 2}} \\
=\frac{a_{0}}{C_{N}^{2^{*}-1}} \frac{1}{\lambda^{N-4}\left|d_{j l}\right|^{N-4}}+O\left(\frac{1}{\lambda^{N-2}\left|d_{j l}\right|^{N-2}}\right) .
\end{gathered}
$$

Let

$$
\begin{gathered}
\Gamma_{1}=\left\{x \in \mathbb{R}^{N}:\left|x-\lambda d_{j l}\right| \leq \frac{\lambda\left|d_{j l}\right|}{4}\right\}, \\
\Gamma_{2}=\left\{x \in \mathbb{R}^{N}:|x| \leq \frac{\lambda\left|d_{j l}\right|}{4}\right\} .
\end{gathered}
$$

We have

$$
\begin{aligned}
& \int_{\mathbb{R}^{N} \backslash\left(\Gamma_{1} \cup \Gamma_{2}\right)} \frac{d x}{\left(1+|x|^{2}\right)^{(N+4) / 2}\left(1+\left|x-\lambda d_{j l}\right|^{2}\right)^{(N-4) / 2}} \\
& \quad \leq \frac{C}{\lambda^{N-4}\left|d_{j l}\right|^{N-4}} \int_{(1 / 4) \lambda\left|d_{j l}\right|}^{+\infty} \frac{\rho^{N-1}}{\left(1+\rho^{2}\right)^{(N+4) / 2}} d \rho \\
& \quad=O\left(\frac{1}{\lambda^{N}\left|d_{j l}\right|^{N}}\right)
\end{aligned}
$$

$$
\begin{aligned}
& \int_{\Gamma_{1}} \frac{d x}{\left(1+|x|^{2}\right)^{(N+4) / 2}\left(1+\left|x-\lambda d_{j l}\right|^{2}\right)^{(N-4) / 2}} \\
& \quad=\int_{0}^{(1 / 4) \lambda\left|d_{j l}\right|} d \rho \\
& \quad \times \int_{\mid x-\lambda d_{j l} l=\rho} \frac{d S}{\left(1+|x|^{2}\right)^{(N+4) / 2}\left(1+\rho^{2}\right)^{(N-4) / 2}} \\
& \leq \frac{C}{\lambda^{N+4}\left|d_{j l}\right|^{N+4}} \int_{0}^{(1 / 4) \lambda\left|d_{j l}\right|} \frac{\rho^{N-1}}{\left(1+\rho^{2}\right)^{(N-4) / 2}} d \rho \\
&=O\left(\frac{1}{\lambda^{N}\left|d_{j l}\right|^{N}}\right) .
\end{aligned}
$$

From (30)-(33), it follows that

$$
\begin{aligned}
\int_{\mathbb{R}^{N}} \frac{d x}{\left(1+|x|^{2}\right)^{(N+4) / 2}\left(1+\left|x-\lambda d_{j l}\right|^{2}\right)^{(N-4) / 2}} \\
=\frac{a_{0}}{C_{N}^{2^{*}-1} \lambda^{N-4}\left|d_{j l}\right|^{N-4}}+O\left(\frac{1}{\lambda^{N-2}\left|d_{j l}\right|^{N-2}}\right) .
\end{aligned}
$$

Hence

$$
\int_{\mathbb{R}^{N}} U_{j}^{2^{*}-1} U_{l} d x=\frac{C_{N} a_{0}}{\lambda^{N-4}\left|d_{j l}\right|^{N-4}}+O\left(\frac{1}{\lambda^{N-2}\left|d_{j l}\right|^{N-2}}\right) .
$$

Combining (23) and (35), we prove

$$
\begin{aligned}
\int_{\Omega} U_{j}^{2^{*}-1} U_{l} d x= & \frac{C_{N} a_{0}}{\left(\lambda\left|d_{j l}\right|\right)^{N-4}} \\
& +O\left(\frac{1}{\left(\lambda\left|d_{j l}\right|\right)^{N-2}}\right)+O\left(\frac{1}{(\lambda r)^{N}}\right) .
\end{aligned}
$$

We denote by $G$ Green's function of $\Delta^{2}$; that is,

$$
\begin{gathered}
\Delta^{2} G(x, \cdot)=2(N-4)(N-2) \omega_{N} \delta_{x} \quad \text { in } \Omega, \\
G(x, \cdot)=\frac{\partial G(x, \cdot)}{\partial \mathbf{n}_{y}}=0 \quad \text { on } \partial \Omega,
\end{gathered}
$$

where $\delta_{x}$ denotes the Dirac mass at $x$, and $\mathbf{n}_{y}$ is the outer unit normal at $y \in \partial \Omega$. We also denote by $H$ the regular part of $G$; that is,

$$
\begin{array}{r}
H(x, y)=|x-y|^{4-N}-G(x, y) \\
\quad \text { for }(x, y) \in \Omega \times \Omega .
\end{array}
$$


By Remark 1 in [16], we have

$$
\begin{aligned}
H(x, y)= & || x\left|y-\frac{x}{|x|}\right|^{4-N} \\
& +\frac{N-4}{2}\left(1-|x|^{2}\right)\left(1-|y|^{2}\right)|| x\left|y-\frac{x}{|x|}\right|^{2-N} .
\end{aligned}
$$

Lemma 3. Consider

$$
\begin{aligned}
& \int_{\Omega} U_{j}^{2^{*}-1} \varphi_{l} d x \\
& \quad= \begin{cases}\frac{C_{N} a_{0}}{\lambda^{N-4}} H\left(x^{(j)}, x^{(j)}\right)+O\left(\lambda^{2-N} r^{1-N}\right), & j=l \\
\frac{C_{N} a_{0}}{\lambda^{N-4}} H\left(x^{(j)}, x^{(l)}\right)+O\left((\lambda r)^{-N}\right), & j \neq l,\end{cases}
\end{aligned}
$$

where $a_{0}=\int_{\mathbb{R}^{N}} U^{2^{*}-1} d x$.

Proof.

Case 1. Consider $j=l$.

Set

$$
\Gamma(x)=\varphi_{j}(x)-C_{N} \lambda^{(4-N) / 2} H\left(x^{(j)}, x\right) .
$$

By the definition of $\varphi_{j}$ and $H\left(x^{(j)}, x\right)$, we get

$$
\Delta^{2} \Gamma(x)=0 \quad \text { in } \Omega,
$$

$$
\Gamma(x)=U_{j}-C_{N} \lambda^{(4-N) / 2}\left|x-x^{(j)}\right|^{4-N} \quad \text { on } \partial \Omega,
$$$$
\frac{\partial \Gamma(x)}{\partial \mathbf{n}}=\frac{\partial U_{j}}{\partial \mathbf{n}}-C_{N} \lambda^{(4-N) / 2} \frac{\partial\left|x-x^{(j)}\right|^{4-N}}{\partial \mathbf{n}} \quad \text { on } \partial \Omega .
$$

For each $x \in \partial \Omega$, we have

$$
\begin{aligned}
\Gamma(x)= & C_{N} \lambda^{(4-N) / 2}\left|x-x^{(j)}\right|^{4-N} \\
& \times\left[\left(1-\frac{1}{1+\lambda^{2}\left|x-x^{(j)}\right|^{2}}\right)^{(N-4) / 2}-1\right] .
\end{aligned}
$$

Thus,

$$
\begin{aligned}
|\Gamma(x)| & \leq C_{N} \lambda^{(4-N) / 2} r^{4-N}\left[1-\left(1-\frac{1}{1+\lambda^{2} r^{2}}\right)^{(N-4) / 2}\right] \\
& \leq C \lambda^{-N / 2} r^{2-N} ;
\end{aligned}
$$

consequently,

$$
\Gamma(x)=O\left(\lambda^{-N / 2} r^{2-N}\right), \quad \text { for } x \in \partial \Omega .
$$

For each $x \in \partial \Omega$, we have

$$
\begin{aligned}
\frac{\partial \Gamma}{\partial \mathbf{n}}(x) & \\
\quad= & C_{N}(4-N)\left(1-x \cdot x^{(j)}\right) \lambda^{(4-N) / 2} \\
& \quad \times\left|x-x^{(j)}\right|^{2-N}\left[\left(1-\frac{1}{1+\lambda^{2}\left|x-x^{(j)}\right|^{2}}\right)^{(N-2) / 2}-1\right] .
\end{aligned}
$$

Since $1-x \cdot x^{(j)} \leq 1-|x| \cdot\left|x^{(j)}\right|=r$, we have

$$
\frac{\partial \Gamma}{\partial \mathbf{n}}(x)=O\left(\lambda^{-N / 2} r^{1-N}\right), \quad \text { for } x \in \partial \Omega .
$$

By [15] (page 155), we have the following explicit formula:

$\Gamma(x)$

$$
\begin{gathered}
=\int_{\partial \Omega} K(x, y)\left(U_{j}(y)-C_{N} \lambda^{(4-N) / 2}\left|y-x^{(j)}\right|^{4-N}\right) d S(y) \\
+C_{N}(N-4) \int_{\partial \Omega} L(x, y)\left(|y|^{2}-y \cdot x^{(j)}\right) \\
\times\left[\lambda^{N / 2}\left(1+\lambda^{2}\left|y-x^{(j)}\right|^{2}\right)^{(2-N) / 2}\right. \\
\left.-\lambda^{(4-N) / 2}\left|y-x^{(j)}\right|^{2-N}\right] d S(y),
\end{gathered}
$$

where

$$
\begin{gathered}
K(x, y) \\
=\frac{1}{2 \omega_{N}} \frac{\left(1-|x|^{2}\right)^{2}}{|x-y|^{N+2}}\left[2+(N-4) x \cdot y-(N-2)|x|^{2}\right], \\
L(x, y)=\frac{1}{2 \omega_{N}} \frac{\left(1-|x|^{2}\right)^{2}}{|x-y|^{N}},
\end{gathered}
$$

with $x \in \Omega, y \in \partial \Omega$. Using (45) and (47), we have, for all $x \in \Omega$,

$$
\begin{aligned}
|\Gamma(x)| \leq & C\left[\lambda^{-N / 2} r^{2-N} \int_{\partial \Omega}|K(x, y)| d S(y)\right. \\
& \left.\quad+\lambda^{-N / 2} r^{1-N} \int_{\partial \Omega} L(x, y) d S(y)\right] \\
\leq & C \lambda^{-N / 2} r^{1-N} \int_{\partial \Omega} L(x, y) d S(y) \\
\leq & C \lambda^{-N / 2} r^{1-N} .
\end{aligned}
$$

Thus,

$$
\Gamma(x)=O\left(\lambda^{-N / 2} r^{1-N}\right), \quad \text { for } x \in \Omega .
$$


We split the term to be estimated as

$$
\begin{aligned}
& \int_{\Omega} U_{j}^{2^{*}-1} \varphi_{j} d x \\
& =C_{N} \lambda^{(4-N) / 2}\left(\int_{B_{r / 2}\left(x^{(j)}\right)}+\int_{\Omega \backslash B_{r / 2}\left(x^{(j)}\right)}\right) \\
& \quad \times U_{j}^{2^{*}-1}(x) H\left(x^{(j)}, x\right) d x \\
& \quad+\int_{\Omega} U_{j}^{2^{*}-1}(x) \Gamma(x) d x
\end{aligned}
$$

and then for the last integral we have

$$
\begin{aligned}
& \left|\int_{\Omega} U_{j}^{2^{*}-1}(x) \Gamma(x) d x\right| \\
& \quad \leq O\left(\lambda^{-N / 2} r^{1-N}\right) \int_{\mathbb{R}^{N}} U_{j}^{2^{*}-1} d x \\
& \quad \leq O\left(\lambda^{-N / 2} r^{1-N}\right) C \lambda^{(4-N) / 2}=O\left(\lambda^{2-N} r^{1-N}\right) .
\end{aligned}
$$

Concerning the integral over $\Omega \backslash B_{r / 2}\left(x^{(j)}\right)$, we first notice that, by (39),

$$
\begin{aligned}
H\left(x^{(j)}, x\right) & \leq r^{4-N}+\frac{N-4}{2} r^{3-N}(2-r)\left(1-|x|^{2}\right) \\
& \leq 2(N-4) r^{3-N} .
\end{aligned}
$$

Therefore,

$$
\begin{aligned}
& C_{N} \lambda^{(4-N) / 2} \int_{\Omega \backslash B_{r / 2}\left(x^{(j)}\right)} U_{j}^{2^{*}-1}(x) H\left(x^{(j)}, x\right) d x \\
& \quad \leq C_{N} \lambda^{(4-N) / 2} 2(N-4) r^{3-N} \int_{\Omega \backslash B_{r / 2}\left(x^{(j)}\right)} U_{j}^{2^{*}-1}(x) d x \\
& \quad=O\left(\lambda^{-N} r^{-1-N}\right) .
\end{aligned}
$$

As in $[5,12]$ we expand $H\left(x^{(j)}, \cdot\right)$ up to the fifth order near $x^{(j)}$, writing

$$
H\left(x^{(j)}, x\right)=H\left(x^{(j)}, x^{(j)}\right)+\sum_{k=1}^{5} H_{k}+R,
$$

where $H_{k}$ denotes the $k$ th order term (e.g., $H_{1}=$ $\left.\nabla H\left(x^{(j)}, x^{(j)}\right)\left(x-x^{(j)}\right)\right)$. Since $|R| \leq \sup _{B_{r / 2}\left(x^{(j)}\right)}\left\|\nabla^{6} H\left(x^{(j)}, \cdot\right)\right\| \cdot$ $\left|x-x^{(j)}\right|^{6}$, using the explicit form of $H$, it is not difficult to check that

$$
|R| \leq \sup _{B_{r / 2}\left(x^{(j)}\right)}\left\|\nabla^{6} H\left(x^{(j)}, \cdot\right)\right\| \leq \frac{C}{r^{N+2}}
$$

so that

$$
\begin{aligned}
& \left|\int_{B_{r / 2}\left(x^{(j)}\right)} U_{j}^{2^{*}-1} R\right| \\
& \quad \leq \frac{C}{r^{N+2}} \int_{B_{r / 2}\left(x^{(j)}\right)} U_{j}^{2^{*}-1}\left|x-x^{(j)}\right|^{6} d x \\
& \quad \leq \frac{C}{\lambda^{(N+4) / 2} r^{N}} .
\end{aligned}
$$

Note that $\Delta^{2} H\left(x^{(j)}, x\right)=0$. Using the symmetry of $U_{j}$ and the usual scaling arguments, we have

$$
\begin{aligned}
& C_{N} \lambda^{(4-N) / 2} \int_{B_{r / 2}\left(x^{(j)}\right)} U_{j}^{2^{*}-1} H\left(x^{(j)}, x\right) d x \\
& \quad \leq C_{N} a_{0} \lambda^{4-N} H\left(x^{(j)}, x^{(j)}\right)+O\left(r^{-N} \lambda^{-N}\right) .
\end{aligned}
$$

By (53)-(59), we obtain

$$
\int_{\Omega} U_{j}^{2^{*}-1} \varphi_{j} d x=\frac{C_{N} a_{0}}{\lambda^{N-4}} H\left(x^{(j)}, x^{(j)}\right)+O\left(\lambda^{2-N} r^{1-N}\right) .
$$

The proof of Lemma 3 is completed.

Case 2. Consider $j \neq l$.

Using the same argument similar to the ones in the proof of the first case, we get the desired result.

Lemma 4. (i) Consider $\int_{B_{j}} U_{j}^{2^{*}} d x=S^{N / 4}+O\left(1 /(\lambda d)^{N}\right)$.

(ii) Consider $\int_{B_{j}} U_{j}^{2^{*}-1} U_{l} d x \geq C_{N} a_{0} /\left(\lambda\left|d_{j l}\right|\right)^{N-4}+$ $O\left(1 /\left(\lambda\left|d_{j l}\right|\right)^{N-2}\right)+O\left(1 / \lambda^{N} d^{4}\left|d_{j l}\right|^{N-4}\right)$.

Proof. (i) The proof makes use of the same estimate as the one in the proof of Lemma 2, with $r$ replaced by $d$ this time.

(ii) We first write

$$
\int_{B_{j}} U_{j}^{2^{*}-1} U_{l} d x=\int_{\mathbb{R}^{N}} U_{j}^{2^{*}-1} U_{l} d x-\int_{\mathbb{R}^{N} \backslash B_{j}} U_{j}^{2^{*}-1} U_{l} d x
$$

and notice that the first integral in the right hand side in (61) has been estimated in (35). Next, we treat the second integral. We will make use of notation and formulas already established in the proof of Lemma 2 to get estimate (35). sition

Since $d / 2 \leq\left|d_{j l}\right| / 4$ by definition, we have the decompo-

$$
\begin{aligned}
\mathbb{R}^{N} \backslash B_{\lambda d / 2}(0)= & \Gamma_{1} \cup\left(\Gamma_{2} \backslash B_{\lambda d / 2}(0)\right) \\
& \cup\left(\mathbb{R}^{N} \backslash\left(\Gamma_{1} \cup \Gamma_{2}\right)\right) .
\end{aligned}
$$

Consequently,

$$
\begin{aligned}
\int_{\mathbb{R}^{N} \backslash B_{j}} & U_{j}^{2^{*}-1} U_{l} d x \\
\leq & \int_{\mathbb{R}^{N} \backslash B_{d / 2}\left(x^{(j)}\right)} U_{j}^{2^{*}-1} U_{l} d x \\
= & C_{N}^{2^{*}}\left(\int_{\Gamma_{1}}+\int_{\Gamma_{2} \backslash B_{\lambda d / 2}(0)}+\int_{\mathbb{R}^{N}\left(\Gamma_{1} \cup \Gamma_{2}\right)}\right) \\
& \times \frac{d x}{\left(1+|x|^{2}\right)^{(N+4) / 2}\left(1+\left|x-\lambda d_{j l}\right|^{2}\right)^{(N-4) / 2}}
\end{aligned}
$$

Now we have to evaluate three integrals in the right hand side in (63). The first integral and the third integral have been estimated in (33) and (32), respectively. Finally, we deal with the second integral over $\Gamma_{2} \backslash B_{\lambda d / 2}(0)$. 
Similar to (27), we have

$$
\begin{gathered}
\int_{\Gamma_{2} \backslash B_{\lambda d / 2}(0)} \frac{d x}{\left(1+|x|^{2}\right)^{(N+4) / 2}\left(1+\left|x-\lambda d_{j l}\right|^{2}\right)^{(N-4) / 2}} \\
\leq \frac{1}{\left(\lambda\left|d_{j l}\right|\right)^{N-4}} \int_{\Gamma_{2} \backslash B_{\lambda d / 2}(0)} \frac{d x}{\left(1+|x|^{2}\right)^{(N+4) / 2}} \\
\quad+\frac{1}{\left(\lambda\left|d_{j l}\right|\right)^{N-2}} \int_{\Gamma_{2} \backslash B_{\lambda d / 2}(0)} \frac{|x|^{2} d x}{\left(1+|x|^{2}\right)^{(N+4) / 2}} .
\end{gathered}
$$

Now with elementary computations we have

$$
\begin{aligned}
& \int_{\Gamma_{2} \backslash B_{\lambda d / 2}(0)} \frac{d x}{\left(1+|x|^{2}\right)^{(N+4) / 2}} \\
& \leq \int_{\mathbb{R}^{N} \backslash B_{\lambda d / 2}(0)} \frac{d x}{\left(1+|x|^{2}\right)^{(N+4) / 2}}=O\left((\lambda d)^{-4}\right) \\
& \int_{\Gamma_{2} \backslash B_{\lambda d / 2}(0)} \frac{|x|^{2} d x}{\left(1+|x|^{2}\right)^{(N+4) / 2}} \\
& \leq \int_{\Gamma_{2}} \frac{|x|^{2} d x}{\left(1+|x|^{2}\right)^{(N+4) / 2}}=\int_{\mathbb{R}^{N}} \frac{|x|^{2} d x}{\left(1+|x|^{2}\right)^{(N+4) / 2}} \\
& \quad+O\left(\left(\lambda\left|d_{j l}\right|\right)^{-2}\right) .
\end{aligned}
$$

Inserting these in (64), we obtain

$$
\begin{aligned}
& \int_{\Gamma_{2} \backslash B_{\lambda d / 2}(0)} \frac{d x}{\left(1+|x|^{2}\right)^{(N+4) / 2}\left(1+\left|x-\lambda d_{j l}\right|^{2}\right)^{(N-4) / 2}} \\
& \quad \leq O\left(\frac{1}{\lambda^{N} d^{4}\left|d_{j l}\right|^{N-4}}\right)+O\left(\frac{1}{\left(\lambda\left|d_{j l}\right|\right)^{N-2}}\right) .
\end{aligned}
$$

Substituting (33), (32), and (66) into (63), we obtain

$$
\begin{aligned}
& \int_{\mathbb{R}^{N} \backslash B_{j}} U_{j}^{2^{*}-1} U_{l} d x \\
& \quad \leq O\left(\frac{1}{\lambda^{N} d^{4}\left|d_{j l}\right|^{N-4}}\right)+O\left(\frac{1}{\left(\lambda\left|d_{j l}\right|\right)^{N-2}}\right) .
\end{aligned}
$$

By (61), (35), and the inequality above, we get the desired estimate.

Lemma 5. Consider

$$
\begin{aligned}
& \int_{B_{j}} U_{j}^{2^{*}-1} \varphi_{l} d x \\
& \quad \leq \begin{cases}\frac{C_{N} a_{0}}{\lambda^{N-4}} H\left(x^{(j)}, x^{(j)}\right)+O\left(\frac{1}{\lambda^{N-1} r^{N}}\right), & j=l, \\
\frac{C_{N} a_{0}}{\lambda^{N-4}} H\left(x^{(j)}, x^{(l)}\right)+O\left(\frac{1}{\lambda^{N-1} d^{N}}\right) & j \neq l,\end{cases}
\end{aligned}
$$

Proof.

Case 1. Consider $j=l$.

Set

$$
\Gamma(x)=\varphi_{j}(x)-C_{N} \lambda^{(N-4) / 2} H\left(x^{(j)}, x\right) .
$$

For $x \in \Omega, y \in \partial \Omega$, we have

$$
\begin{aligned}
2+ & (N-4) x \cdot y-(N-2)|x|^{2} \\
& \leq 2+(N-4)|x|-(N-2)|x|^{2}<0 .
\end{aligned}
$$

By definition of $K(x, y)$, we get

$$
K(x, y)<0 \quad \text { for } x \in \Omega, y \in \partial \Omega \text {. }
$$

By (48), we obtain

$$
\Gamma(x)<0 \quad \text { for } x \in \Omega
$$

Therefore, we can write

$$
\begin{aligned}
& \int_{B_{j}} U_{j}^{2^{*}-1} \varphi_{j} d x \\
& \quad=\frac{C_{N}}{\lambda^{N-4}} \int_{B_{j}} U_{j}^{2^{*}-1} H\left(x^{(j)}, x\right) d x+\int_{B_{j}} U_{j}^{2^{*}-1} \Gamma d x \\
& \quad \leq \frac{C_{N}}{\lambda^{N-4}} \int_{B_{j}} U_{j}^{2^{*}-1} H\left(x^{(j)}, x\right) d x .
\end{aligned}
$$

Since $d \leq(1 / 2) r$, the last term can be estimated as in (59); namely,

$$
\begin{aligned}
& \frac{C_{N}}{\lambda^{(N-4) / 2}} \int_{B_{j}} U_{j}^{2^{*}-1} H\left(x^{(j)}, x\right) d x \\
& \quad \leq \frac{C_{N}}{\lambda^{(N-4) / 2}} \int_{B_{r / 2}\left(x^{(j)}\right)} U_{j}^{2^{*}-1} H\left(x^{(j)}, x\right) d x \\
& \quad \leq \frac{C_{N} a_{0}}{\lambda^{N-4}} H\left(x^{(j)}, x^{(j)}\right)+O\left(r^{-N} \lambda^{1-N}\right),
\end{aligned}
$$

which gives the required estimate.

Case 2. Consider $j \neq l$.

The computation can be adapted from the ones in the proof of Case 1.

Define

$$
\widetilde{u}(x)=\sum_{j=0}^{k-1} P U_{j}=\sum_{j=0}^{k-1}\left(U_{j}-\varphi_{j}\right) .
$$

Due to the definition of the points $x^{(j)}$, we have $\tilde{u} \in H_{k}$. Notice that $\tilde{u}$ depends on $\lambda, k$, and $r$ through the choice of the points $x^{(j)}$. 
Lemma 6. As $\lambda d \rightarrow \infty$ (i.e., $\lambda \rightarrow 0$ ), one has

$$
\begin{aligned}
& \int_{\Omega}|\Delta \tilde{u}|^{2} d x \\
& =k S^{N / 4}-\frac{C_{N} a_{0}}{\lambda^{N-4}} \\
& \times\left\{\sum_{j=0}^{k-1} H\left(x^{(j)}, x^{(j)}\right)-\sum_{\substack{j, l=0 \\
j \neq l}}^{k-1} G\left(x^{(j)}, x^{(j)}\right)\right\} \\
& +k^{N-1} O\left(\lambda^{2-N}\right)+k^{2} O\left((\lambda r)^{-N}\right), \\
& \int_{\Omega}|x|^{\alpha} \widetilde{u}^{2^{*}} d x \\
& \geq(1-2 r)^{\alpha} \\
& \times\left[k S^{N / 4}-\frac{2^{*} C_{N} a_{0}}{\lambda^{N-4}}\right. \\
& \times\left\{\sum_{j=0}^{k-1} H\left(x^{(j)}, x^{(j)}\right)-\sum_{\substack{j, l=0 \\
j \neq l}}^{k-1} G\left(x^{(j)}, x^{(j)}\right)\right\} \\
& \left.+k^{N-1} O\left(\frac{1}{\lambda^{N-2}}\right)+k^{2} O\left(\frac{1}{\lambda^{N-1} r^{N}}\right)\right] \text {. }
\end{aligned}
$$

Proof. By definition of $u$ (see (75)) and $P U_{j}$,

$$
\begin{aligned}
\int_{\Omega}|\Delta \tilde{u}|^{2} d x \\
=\sum_{j, l=0}^{k-1}\left(\int_{\Omega} U_{j}^{2^{*}} d x-\int_{\Omega} U_{j}^{2^{*}-1} \varphi_{j} d x\right) \\
\quad+\sum_{\substack{j, l=0 \\
j \neq l}}^{k-1}\left(\int_{\Omega} U_{j}^{2^{*}-1} U_{l} d x-\int_{\Omega} U_{j}^{2^{*}-1} \varphi_{l} d x\right) .
\end{aligned}
$$

By Lemmas 2 and 3, we have

$$
\begin{aligned}
& \sum_{j=0}^{k-1}\left(\int_{\Omega} U_{j}^{2^{*}} d x-\int_{\Omega} U_{j}^{2^{*}-1} \varphi_{j} d x\right) \\
& =k S^{N / 4}-\frac{C_{N} a_{0}}{\lambda^{N-4}} \sum_{j=0}^{k-1} H\left(x^{(j)}, x^{(j)}\right) \\
& +k O\left((\lambda r)^{-N}\right), \sum_{\substack{j, l=0 \\
j \neq l}}^{k-1} \int_{\Omega} U_{j}^{2^{*}-1} \varphi_{l} d x \\
& =\frac{C_{N} a_{0}}{\lambda^{N-4}} \sum_{\substack{j, l=0 \\
j \neq l}}^{k-1}\left(x^{(j)}, x^{(l)}\right) \\
& +\left(k^{2}-k\right) O\left((\lambda r)^{-N}\right) .
\end{aligned}
$$

By the symmetry of the points $x^{(j)}$, we have

$$
\begin{aligned}
\sum_{\substack{j, l=0 \\
j \neq l}}^{k-1} \frac{1}{\left|d_{j l}\right|^{N-2}} & =k \sum_{j=1}^{k-1} \frac{1}{(2-2 r)^{N-2} \sin ^{N-2}(\pi j / k)} \\
& \sim \frac{2 k}{(2-2 r)^{N-2}} \sum_{j=1}^{[(k-1) / 2]} \frac{C}{(j / k)^{N-2}} \\
& \sim C k^{N-1},
\end{aligned}
$$

since the series of $j^{1-N}$ is convergent. Recall that $r$ will be taken small so that we can always assume $r \leq 1 / 2$. By (80), we obtain from Lemma 2

$$
\begin{aligned}
& \sum_{\substack{j, l=0 \\
j \neq l}}^{k-1} \int_{\Omega} U_{j}^{2^{*}-1} U_{l} d x \\
& \quad=\frac{C_{N} a_{0}}{\lambda^{N-4}} \sum_{\substack{j, l=0 \\
j \neq l}}^{k-1} \frac{1}{\left|x^{(j)}-x^{(l)}\right|^{N-4}} \\
& \quad+k^{N-1} O\left(\lambda^{2-N}\right)+\left(k^{2}-k\right) O\left((\lambda r)^{-N}\right) .
\end{aligned}
$$

Substituting (79) and (81) into (78) and recalling the definition of $G$, we prove (76).

Having completed the estimate of the numerator of $Q_{\alpha}$, we now go on to estimate the denominator, namely, $\int_{\Omega}|x|^{\alpha} \tilde{u}^{2^{*}} d x$. Recall that we denote $d=(1 / 2) \min _{j \neq l} \mid x^{(j)}-$ $x^{(l)} \mid$ and that we assume $2 d \leq r$. We now set $B_{j}=B_{d}\left(x^{(j)}\right)$ for $j=0,1, \ldots, k-1$. Then the $B_{j}$ 's are positive disjoint and they are all contained in $\Omega \backslash \bar{B}_{1-2 r}(0)$. Hence

$$
\begin{aligned}
\int_{\Omega}|x|^{\alpha} \widetilde{u}^{2^{*}} d x & \geq(1-2 r)^{\alpha} \int_{\Omega \backslash \bar{B}_{1-2 r}(0)} \widetilde{u}^{2^{*}} d x \\
& \geq(1-2 r)^{\alpha} \sum_{j=0}^{k-1} \int_{B_{j}} \tilde{u}^{2^{*}} d x .
\end{aligned}
$$

It is easy to see that

$$
\begin{aligned}
\tilde{u}^{2^{*}} & =\left(\sum_{l=0}^{k-1} P U_{l}\right)^{2^{*}} \\
& =\left[U_{j}+\sum_{\substack{l=0 \\
l \neq j}}^{k-1}\left(U_{l}-\varphi_{l}\right)-\varphi_{j}\right]^{2^{*}} \\
& \geq U_{j}^{2^{*}}+2^{*} U_{j}^{2^{*}-1}\left[\sum_{\substack{l=0 \\
l \neq j}}^{k-1}\left(U_{l}-\varphi_{l}\right)-\varphi_{j}\right]^{2^{*}},
\end{aligned}
$$

where we have used the inequality

$$
(a+b)^{p} \geq a^{p}+p a^{p-1} b,
$$


for $p \geq 1, a \geq 0, a+b \geq 0$. We obtain therefore

$$
\begin{aligned}
& \sum_{j=0}^{k-1} \int_{B_{j}} \widetilde{u}^{2^{*}} d x \\
& \geq \sum_{j=0}^{k-1}\left(\int_{B_{j}} U_{j}^{2^{*}} d x-2^{*} \int_{B_{j}} U_{j}^{2^{*}-1} \varphi_{j} d x\right) \\
& \quad+2^{*} \sum_{\substack{j, l=0 \\
j \neq l}}^{k-1}\left(\int_{B_{j}} U_{j}^{2^{*}-1} U_{l} d x-\int_{B_{j}} U_{j}^{2^{*}-1} \varphi_{l} d x\right),
\end{aligned}
$$

and we estimate the four integrals separately as above.

By the first part of Lemmas 4 and 5, and recalling that $r>d \sim C / k$ and

$$
\begin{aligned}
& \sum_{j=0}^{k-1}\left(\int_{B_{j}} U_{j}^{2^{*}} d x-2^{*} \int_{B_{j}} U_{j}^{2^{*}-1} \varphi_{j} d x\right) \\
& \geq k S^{N / 4}-\frac{2^{*} C_{N} a_{0}}{\lambda^{N-4}} \sum_{j=0}^{k-1} H\left(x^{(j)}, x^{(j)}\right) \\
& \quad+k^{N} O\left(\frac{1}{\lambda^{N-1}}\right) .
\end{aligned}
$$

The remainders generated by the second part of Lemma 4 can be dealt with as in (80). We obtain

$$
\begin{aligned}
& \sum_{\substack{j, l=0 \\
j \neq l}}^{k-1} \frac{1}{\left|d_{j l}\right|^{N-2}} \sim C k^{N-1}, \\
& \sum_{\substack{j, l=0 \\
j \neq l}}^{k-1} \frac{1}{\left|d_{j l}\right|^{N-4}} \sim C k^{N-3} .
\end{aligned}
$$

Therefore,

$$
\begin{aligned}
2^{*} \sum_{\substack{j, l=0 \\
j \neq l}}^{k-1} \int_{B_{j}} U_{j}^{2^{*}-1} U_{l} d x \geq & \frac{2^{*} C_{N} a_{0}}{\lambda^{N-4}} \sum_{\substack{j, l=0 \\
j \neq l}}^{k-1} \frac{1}{\left|x^{(j)}-x^{(l)}\right|^{N-4}} \\
& +k^{N-1} O\left(\frac{1}{\lambda^{N-2}}\right)+k^{N+1} O\left(\frac{1}{\lambda^{N}}\right) \\
= & \frac{2^{*} C_{N} a_{0}}{\lambda^{N-4}} \sum_{\substack{j, l=0 \\
j \neq l}}^{k-1} \frac{1}{\left|x^{(j)}-x^{(l)}\right|^{N-4}} \\
& +k^{N-1} O\left(\frac{1}{\lambda^{N-2}}\right)
\end{aligned}
$$

since $k / \lambda \rightarrow 0$.

Finally, from Lemma 5,

$$
\begin{aligned}
2^{*} \sum_{\substack{j, l=0 \\
j \neq l}}^{k-1} \int_{B_{j}} U_{j}^{2^{*}-1} \varphi_{l} d x \leq & \frac{2^{*} C_{N} a_{0}}{\lambda^{N-4}} \sum_{\substack{j, l=0 \\
j \neq l}}^{k-1} H\left(x^{(j)}, x^{(l)}\right) \\
& +\left(k^{2}-k\right) O\left(\frac{1}{\lambda^{N-1} r^{N}}\right) .
\end{aligned}
$$

Substituting (86), (88), and (89) into (85) and recalling the definition of $G$, we obtain the required estimate.

Proposition 7. Let $N \geq 6$. For every $\alpha>0$, there exists $k_{\alpha}>0$ such that, for every integer $k \geq k_{\alpha}$,

$$
\Sigma_{k}<k^{4 / N} S \text {. }
$$

Proof. The function $\tilde{u}$ constructed in (75) depends on $k, r$, and $\lambda$, and for each $k$ it belongs to $H_{k}$. We show that, for appropriate choice of these parameters, there results $Q_{\alpha}(\widetilde{u})<$ $k^{4 / N} S$. For simplicity, we set

$$
A:=\sum_{j=0}^{k-1} H\left(x^{(j)}, x^{(j)}\right)-\sum_{\substack{j, l=0 \\ j \neq l}}^{k-1} G\left(x^{(j)}, x^{(l)}\right)
$$

and we begin with an estimate of $A$, noticing that we can write it as

$$
A=\sum_{j, l=0}^{k-1} H\left(x^{(j)}, x^{(l)}\right)-\sum_{\substack{j, l=0 \\ j \neq l}}^{k-1} \frac{1}{\left|x^{(j)}-x^{(l)}\right|^{N-4}} .
$$

By definition of $H$ and since $\left.\left|x^{(j)}-x^{(l)} /\right| x^{(l)}\right|^{2} \mid \geq r$ for all $j, l$, we have

$$
\begin{aligned}
& \sum_{j, l=0}^{k-1} H\left(x^{(j)}, x^{(l)}\right) \\
& \quad \leq \sum_{j, l-0}^{k-1}\left(\frac{1}{\left|x^{(j)}\right|^{N-4} r^{N-4}}+\frac{1}{\left|x^{(j)}\right|^{N-2} r^{N-2}}\right) \\
& \quad \leq \frac{C_{1} k^{2}}{r^{N-2}} .
\end{aligned}
$$

Moreover, as in (87),

$$
\sum_{\substack{j, l=0 \\ j \neq l}}^{k-1} \frac{1}{\left|x^{(j)}-x^{(l)}\right|^{N-4}} \sim C_{2} k^{N-3},
$$

so we obtain

$$
A \leq \frac{C_{1} k^{2}}{r^{N-2}}-C_{2} k^{N-3} .
$$

Note that $(1-2 r)^{2 \alpha / 2^{*}} \geq 1-3 \alpha r$, for all $N \geq 6, \alpha>0$ and $r$ small enough; we see that, from Lemma 6,

$$
\begin{aligned}
& \left(\int_{\Omega}|x|^{\alpha} \widetilde{u}^{2^{*}} d x\right)^{2 / 2^{*}} \\
& \geq(1-3 \alpha r) k^{2 / 2^{*}} \\
& \quad \times\left[S^{N / 4}-\frac{2^{*} C_{N} a_{0}}{k \lambda^{N-4}} A\right. \\
& \left.\quad+k^{N-2} O\left(\frac{1}{\lambda^{N-2}}\right)+k O\left(\frac{1}{\lambda^{N-1} r^{N}}\right)\right]^{2 / 2^{*}} .
\end{aligned}
$$


Choose $r=k^{-3(N-5) / 4(N-2)}$ and $\lambda=k^{1+\varepsilon}$ with $\varepsilon>0$ and small. It is easy to see that all the quantities depending on $k$ in the square brackets tend to zero as $\lambda / k \rightarrow \infty$; therefore, we obtain

$Q_{\alpha}(\widetilde{u})$

$$
\begin{gathered}
\leq\left(k S^{N / 4}-\frac{C_{N} a_{0}}{\lambda^{N-4}} A+k^{N-1} O\left(\frac{1}{\lambda^{N-2}}\right)+k^{2} O\left(\frac{1}{(\lambda r)^{N}}\right)\right) \\
\times\left((1-3 \alpha r) k^{2 / 2^{*}}\right. \\
\times\left[S^{(N-4) / 4}-\frac{2 C_{N} a_{0}}{k S \lambda^{N-4}} A\right. \\
\left.\left.\quad+k^{N-2} O\left(\frac{1}{\lambda^{N-2}}\right)+k O\left(\frac{1}{\lambda^{N-1} r^{N}}\right)\right]\right)^{-1} .
\end{gathered}
$$

We must check that, for suitable values of the parameters, the right hand side is strictly less than $k^{4 / N} S$. Direct computations show that it is enough to prove

$$
\begin{aligned}
R:= & 3 \alpha r S^{N / 4}+(1-6 \alpha r) \frac{C_{N} a_{0}}{k \lambda^{N-4}} A+k^{N-2} O\left(\frac{1}{\lambda^{N-2}}\right) \\
& -(1-3 \alpha r) S k^{N-2} O\left(\frac{1}{\lambda^{N-2}}\right) \\
& +k^{2}\left(\frac{1}{(\lambda r)^{N}}\right)-(1-3 \alpha r) S k O\left(\frac{1}{\lambda^{N-1} r^{N}}\right)<0 .
\end{aligned}
$$

We take $k$ so large that

$$
A \leq-\frac{C_{2}}{2} k^{N-3},
$$

and this is possible because

$$
A \leq \frac{C_{1} k^{2}}{r^{N-2}}-C_{2} k^{N-3}=C_{1} k^{2+(3 / 4)(N-5)}-C_{2} k^{N-3}
$$

and $2+(3 / 4)(N-5)<N-3$ for all $N \geq 6$, as one immediately checks. Furthermore, noticing that $3 N(N-5) / 4(N-2)<$ $N-2$, we see that

$$
\begin{gathered}
\frac{k^{2}}{(\lambda r)^{N}}=\frac{k^{2+3 N(N-5) / 4(N-2)}}{\lambda^{N}} \leq \frac{k^{N}}{\lambda^{N}} \leq \frac{k^{N-2}}{\lambda^{N-2}}, \\
\frac{k}{\lambda^{N-1} r^{N}}=\frac{k^{1+3 N(N-5) / 4(N-2)}}{\lambda^{N-1}} \leq \frac{k^{N-1}}{\lambda^{N-1}} \leq \frac{k^{N-2}}{\lambda^{N-2}},
\end{gathered}
$$

since $k / \lambda \rightarrow 0$. Therefore, the third and the last big $O$ is unnecessary in the expression of $R$. We are thus led to

$$
\begin{aligned}
R \leq & \frac{3 \alpha S^{N / 4}}{k^{3(N-5) / 4(N-2)}}-\left(1-\frac{6 \alpha}{k^{3(N-5) / 4(N-2)}}\right) \\
& \cdot \frac{C_{N} a_{0} C_{2}}{2} \cdot \frac{k^{N-4}}{\lambda^{N-4}}+k^{N-2} O\left(\frac{1}{\lambda^{N-2}}\right) \\
= & \frac{3 \alpha S^{N / 4}}{k^{3(N-5) / 4(N-2)}}-\left(1-\frac{6 \alpha}{k^{3(N-5) / 4(N-2)}}\right) \\
& \cdot \frac{C_{N} a_{0} C_{2}}{2} \cdot \frac{1}{k^{\varepsilon(N-4)}}+O\left(\frac{1}{k^{\varepsilon(N-2)}}\right) .
\end{aligned}
$$

Since $\alpha$ is fixed, we have $R<0$ for $k$ large (depending on $\alpha$ ) if we take $\varepsilon$ small enough (essentially $\varepsilon<3(N-5) / 4(N-2)(N-$ 4)).

Next we show that if $\Sigma_{k}<k^{4 / N} S$ then $\Sigma_{k}$ is achieved. So we are led to analyze what happens if a minimizing sequence in $H_{k}$ tends weakly to zero in $H_{0}^{2}(\Omega)$. Let $u_{n} \in H_{k}$ be a minimizing sequence for problem (9) such that $u_{n} \rightarrow 0$ weakly in $H_{0}^{2}(\Omega)$. Without loss of generality, we can assume that $Q_{\alpha}^{\prime}\left(u_{n}\right) \rightarrow 0$ in $H_{k}$ by Ekeland's variational principle.

Since $Q_{\alpha}$ is invariant under the action of $G_{k}$, we also have $Q_{\alpha}^{\prime}\left(u_{n}\right) \rightarrow 0$ in $H_{0}^{2}(\Omega)$. By homogeneity of $Q_{\alpha}(u)$, we normalize $u_{n}$ to obtain a sequence (still denoted by $u_{n}$ ) such that as $n \rightarrow \infty$,

$$
\begin{gathered}
Q_{\alpha}\left(u_{n}\right) \longrightarrow \Sigma_{k}, \quad Q_{\alpha}^{\prime}\left(u_{n}\right) \longrightarrow 0 \quad \text { in } H_{0}^{2}(\Omega), \\
u_{n} \rightarrow 0 \quad \text { in } H_{0}^{2}(\Omega) \\
\int_{\Omega}|x|^{\alpha}\left|u_{n}\right|^{2^{*}} d x=\Sigma_{k}^{N / 4} \\
\int_{\Omega}\left|\Delta u_{n}\right|^{2} d x=\Sigma_{k}^{N / 4}+o(1)
\end{gathered}
$$

The corresponding energy functional of problem (1) is defined by

$$
\begin{array}{r}
J_{\alpha}(u)=\frac{1}{2} \int_{\Omega}|\Delta u|^{2} d x-\frac{1}{2} \int_{\Omega}|x|^{\alpha}|u|^{2^{*}} d x, \\
u \in H_{0}^{2}(\Omega) .
\end{array}
$$

By (103), direct computation shows that

$$
\begin{aligned}
& J_{\alpha}\left(u_{n}\right) \longrightarrow \frac{2}{N} \Sigma_{k}^{N / 4}, J_{\alpha}^{\prime}\left(u_{n}\right) \longrightarrow 0 \\
& \text { as } n \longrightarrow \infty .
\end{aligned}
$$

Thus, $\left\{u_{n}\right\}$ is a Palais-Smale sequence for the functional $J_{\alpha}$ at level $(2 / N) \Sigma_{k}^{N / 4}$.

Since for all $n$

$$
\begin{aligned}
& \int_{\mathbb{R}^{N}}\left|u_{n}\right|^{2^{*}} d x \\
& \quad=\int_{\Omega}\left|u_{n}\right|^{2^{*}} d x \geq \int_{\Omega}|x|^{\alpha}\left|u_{n}\right|^{2^{*}} d x=\Sigma_{k}^{N / 4}>0,
\end{aligned}
$$

by Lemma 10.1 in [17], there exists a sequence of rescaling $\mathscr{T}_{n}=\mathscr{T}\left(\lambda_{n}, q_{n}\right)$ such that

$$
\mathscr{T}_{n} u_{n} \rightarrow u \quad \text { weakly in } D^{2,2}\left(\mathbb{R}^{N}\right), u \neq \equiv .
$$

Since $\operatorname{supp} u_{n} \subset \Omega$, we get $\lambda_{n} \rightarrow \infty$ as $n \rightarrow \infty$ and $q_{n} \in \bar{\Omega}$. We can also assume that $q_{n} \rightarrow q \in \bar{\Omega}$.

Lemma 8. Let $\mathscr{T}_{n}=\mathscr{T}\left(\lambda_{n}, q_{n}\right)$ be the above sequence of rescaling satisfying $\lambda \rightarrow \infty, q_{n} \rightarrow q \in \bar{\Omega}$, and $\mathscr{T}_{n} u_{n} \rightarrow u \neq \equiv$ 0 weakly in $D^{2,2}\left(\mathbb{R}^{N}\right)$. Then $\lambda_{n} \operatorname{dist}\left(q_{n}, \partial \Omega\right) \rightarrow \infty, q \neq 0$, and u satisfies

$$
\Delta^{2} u=|q|^{\alpha}|u|^{2^{*}-2} u \quad \text { in } \mathbb{R}^{N} .
$$


Proof. We first prove $\lambda_{n} \operatorname{dist}\left(q_{n}, \partial \Omega\right) \rightarrow \infty$ as $n \rightarrow \infty$. Assume to the contrary that $\lambda_{n} \operatorname{dist}\left(q_{n}, \partial \Omega\right) \leq C<\infty$ for all $n$. Considering $\Omega_{n}=\left\{x \in \mathbb{R}^{N}: x / \lambda_{n}+q_{n} \in \Omega\right\}$, we may regard $\mathscr{T}_{n} u_{n} \in H_{0}^{2}\left(\Omega_{n}\right) \subset D^{2,2}\left(\mathbb{R}^{N}\right)$. After a rotation of coordinates, we may assume that the sequence $\left\{\Omega_{n}\right\}$ exhausts the half space $\mathbb{R}_{+}^{N}$. For any $\psi \in C_{0}^{\infty}\left(\mathbb{R}_{+}^{N}\right)$, we have $\psi \in$ $C_{0}^{\infty}\left(\Omega_{n}\right)$ for all large $n$. Thus, the support of $\mathscr{T}_{n}^{-1} \psi$ is contained in $\Omega$ for all large $n$.

Set

$$
\begin{aligned}
& \phi(x)= \begin{cases}|x|^{\alpha} & x \in \bar{\Omega}, \\
0, & x \notin \bar{\Omega},\end{cases} \\
& \phi_{n}(x)=\phi\left(\frac{x}{\lambda_{n}}+q_{n}\right) .
\end{aligned}
$$

The fact that $\left\{u_{n}\right\}$ is a Palais-Smale sequence for $J_{\alpha}$ implies that

$$
\begin{aligned}
o(1) & =\left\langle J_{\alpha}^{\prime}\left(u_{n}\right), \mathscr{T}_{n}^{-1} \psi\right\rangle \\
& =\int_{\mathbb{R}^{N}} \Delta u \Delta \psi d x-\int_{\mathbb{R}^{N}} \phi(q) u^{2^{*}-1} \psi d x+o(1) .
\end{aligned}
$$

We have used the fact that $\mathscr{T}_{n} u_{n} \rightarrow u$ in $D^{2,2}\left(\mathbb{R}^{N}\right)$ and $\phi_{n}(x) \rightarrow \phi(q)$. Since this happens for all $\psi \in C_{0}^{\infty}\left(\mathbb{R}_{+}^{\mathbb{N}}\right)$, we see that $u$ satisfies

$$
\begin{gathered}
\Delta^{2} u=|q|^{\alpha} u^{2^{*}-1}, \quad u \in D^{2,2}\left(\mathbb{R}^{N}\right) \text { in } \Pi, \\
u=\frac{\partial u}{\partial x_{N}}=0 \quad \text { on } \partial \Pi,
\end{gathered}
$$

where $\Pi=\left\{\left(x^{\prime}, x_{N}\right) \in \mathbb{R}^{N}: x_{N} \geq-L\right\}$ for some constant $L \geq$ 0 . By Lemma 4 in [2] (also see [4]), (111) has a unique solution $u \equiv 0$, which contradicts $u \neq \equiv 0$. Therefore, we must have $\lambda_{n} \operatorname{dist}\left(q_{n}, \partial \Omega\right) \rightarrow 0$. In this case, we can repeat the above argument and take test function $\psi \in C_{0}^{\infty}\left(\mathbb{R}^{N}\right)$. The above computations imply that $u$ is a nontrivial solution of $\Delta^{2} u=$ $|q|^{\alpha}|u|^{2^{*}-2} u$ in $\mathbb{R}^{N}$, which also shows that $q \neq 0$ since $u \in$ $D^{2,2}\left(\mathbb{R}^{N}\right)$.

Remark 9. It is proved in [18] that the radial function

$$
U(x)=C_{N}\left(1+|x|^{2}\right)^{-(N-4) / 2}
$$

solves $\Delta^{2} U=U^{2^{*}-1}$ in $\mathbb{R}^{N}$, where $C_{N}=[(N-4)(N-2) N(N+$ $2)]^{(N-4) / 8}$. So the function $u$ is nothing else than a multiple of $U$. Precisely, $u=|q|^{\alpha(4-N) / 8} U$.

Let $\chi \in C_{0}^{\infty}\left(\mathbb{R}^{N}\right)$ be a cut-off function satisfying $0 \leq$ $\chi(x) \leq 1$ for all $x \in \mathbb{R}^{N}, \chi(x)=1$ for $|x| \leq 1, \chi(x)=0$ for $|x| \geq 2,|\nabla \chi| \leq C$, and $|\Delta \chi| \leq C$.

Since $\lambda_{n} \operatorname{dist}\left(q_{n}, \partial \Omega\right) \rightarrow \infty$, we may take a sequence $\left\{\bar{\lambda}_{n}\right\}$ such that $\bar{\lambda}_{n}>0, \bar{\lambda}_{n} \rightarrow \infty, \lambda_{n} / \bar{\lambda}_{n} \rightarrow \infty$ and $\bar{\lambda}_{n} \operatorname{dist}\left(q_{n}, \partial \Omega\right) \rightarrow \infty$ as $n \rightarrow \infty$. Set $\chi_{n}(x)=\chi\left(\left(\bar{\lambda}_{n} / \lambda_{n}\right) x\right)$. In this way, supp $\mathscr{T}_{n}^{-1} \chi_{n} \subset \Omega$ for all $n$ large and $u_{n}-$ $\mathscr{T}_{n}^{-1}\left(\chi_{n} u\right) \in H_{0}^{2}(\Omega)$.
Note that

$$
\begin{aligned}
\int_{\mathbb{R}^{N}}\left|\Delta\left(\chi_{n} u-u\right)\right|^{2} d x & \\
\leq C & \left(\int_{\mathbb{R}^{N} \backslash B_{\lambda_{n} / \bar{\lambda}_{n}}(0)}|\Delta u|^{2} d x\right. \\
& +\left(\frac{\bar{\lambda}_{n}}{\lambda_{n}}\right)^{2} \int_{B_{2 \lambda_{n} / \bar{\lambda}_{n}}(0) \backslash B_{\lambda_{n} / \bar{\lambda}_{n}}(0)}|\nabla u|^{2} d x \\
& \left.+\left(\frac{\bar{\lambda}_{n}}{\lambda_{n}}\right)^{4} \int_{B_{2 \lambda_{n} / \bar{\lambda}_{n}}(0) \backslash B_{\lambda_{n} / \bar{\lambda}_{n}}(0)}|u|^{2} d x\right) .
\end{aligned}
$$

Since $\Delta u \in L^{2}\left(\mathbb{R}^{N}\right)$, the first term tends to 0 as $n \rightarrow \infty$. By Hölder inequality, the second term and the third term in inequality above,

$$
\begin{aligned}
& \left(\frac{\bar{\lambda}_{n}}{\lambda_{n}}\right)^{2} \int_{B_{2 \lambda_{n} / \bar{\lambda}_{n}}(0) \backslash B_{\lambda_{n} / \bar{\lambda}_{n}}(0)}|\nabla u|^{2} d x \\
& \leq C\left(\frac{\bar{\lambda}_{n}}{\lambda_{n}}\right)^{2} \\
& \quad \times\left(\int_{B_{2 \lambda_{n} / \bar{\lambda}_{n}}(0) \backslash B_{\lambda_{n} / \bar{\lambda}_{n}}(0)}|\nabla u|^{2 N /(N-2)} d x\right)^{(N-2) / N} \longrightarrow 0, \\
& \left(\frac{\bar{\lambda}_{n}}{\lambda_{n}}\right)^{4} \int_{B_{2 \lambda_{n} / \bar{\lambda}_{n}}(0) \backslash B_{\lambda_{n} / \bar{\lambda}_{n}}(0)}|u|^{2} d x \\
& \leq C\left(\frac{\bar{\lambda}_{n}}{\lambda_{n}}\right)^{4}\left(\int_{B_{2 \lambda_{n} / \bar{\lambda}_{n}}(0) \backslash B_{\lambda_{n} / \bar{\lambda}_{n}}(0)}|u|^{2^{*}} d x\right)^{2 / 2^{*}} \longrightarrow 0
\end{aligned}
$$

as $n \rightarrow \infty$. Thus, $\chi_{n} u \rightarrow u$ strongly in $D^{2,2}\left(\mathbb{R}^{N}\right)$.

Lemma 10. Let $\mathscr{T}_{n} u_{n}$ be the sequence constructed above. Then, as $n \rightarrow \infty$, one has

(i) $J_{\alpha}\left(u_{n}-\mathscr{T}_{n}^{-1}\left(\chi_{n} u\right)\right)=J_{\alpha}\left(u_{n}\right)-J_{\alpha, q}(u)+o(1)$,

(ii) $J_{\alpha}^{\prime}\left(u_{n}-\mathscr{T}_{n}^{-1}\left(\chi_{n} u\right)\right)=J_{\alpha}^{\prime}\left(u_{n}\right)+o(1)$ in $H_{0}^{2}(\Omega)$,

where

$$
J_{\alpha, q}(u)=\frac{1}{2} \int_{\mathbb{R}^{N}}|\Delta u|^{2} d x-\frac{1}{2^{*}}|q|^{\alpha} \int_{\mathbb{R}^{N}}|u|^{2^{*}} d x .
$$

Moreover, the sequence $\left\{u_{n}-\mathscr{T}_{n}^{-1}\left(\chi_{n} u\right)\right\}$ is a Palais-Smale sequence for $J_{\alpha}$ at level $(2 / N) \Sigma_{k}^{N / 4}-J_{\alpha, q}(u)$; namely, it satisfies

(i) $J_{\alpha}\left(u_{n}-\mathscr{T}_{n}^{-1}\left(\chi_{n} u\right)\right)=(2 / N) \Sigma^{N / 4}-J_{\alpha, q}(u)+o(1)$;

(ii) $J_{\alpha}^{\prime}\left(u_{n}-\mathscr{T}_{n}^{-1}\left(\chi_{n} u\right)\right)=o(1)$ in $H_{0}^{2}(\Omega)$.

Proof. (i) Set $w_{n}=u_{n}-\mathscr{T}_{n}^{-1}\left(\chi_{n} u\right)$; then $w_{n} \in H_{0}^{2}(\Omega)$. Since $\mathscr{T}_{n} u_{n} \rightarrow u$ and $\chi_{n} u \rightarrow u$ in $D^{2,2}\left(\mathbb{R}^{N}\right)$, we have

$$
\int_{\Omega}\left|\Delta w_{n}\right|^{2} d x=\int_{\mathbb{R}^{N}}\left|\Delta u_{n}\right|^{2} d x-\int_{\mathbb{R}^{N}}|\Delta u|^{2} d x+o(1) .
$$


Set $\phi_{n}(x)=\phi\left(x / \lambda_{n}+q_{n}\right)$. Changing variables as in the first part, we have

$$
\begin{aligned}
\int_{\Omega}|x|^{\alpha}\left|w_{n}\right|^{2^{*}} d x & =\int_{\mathbb{R}^{N}} \phi(x)\left|w_{n}\right|^{2^{*}} d x \\
& =\int_{\mathbb{R}^{N}} \phi_{n}(x)\left|\mathscr{T}_{n} u_{n}-\chi_{n} u\right|^{2^{*}} d x .
\end{aligned}
$$

Since $\chi_{n} u \rightarrow u$ in $D^{2,2}\left(\mathbb{R}^{N}\right)$, we get by the Brézis-Lieb lemma [19]:

$$
\begin{aligned}
\int_{\mathbb{R}^{N}} & \phi_{n}(x)\left|\mathscr{T}_{n} u_{n}-\chi_{n} u\right|^{2^{*}} d x \\
= & \int_{\mathbb{R}^{N}} \phi_{n}(x)\left|\mathscr{T}_{n} u_{n}-u\right|^{2^{*}} d x+o(1) \\
= & \int_{\mathbb{R}^{N}} \phi_{n}(x)\left|\mathscr{T}_{n} u_{n}\right|^{2^{*}} d x \\
& -\int_{\mathbb{R}^{N}} \phi_{n}(x)|u|^{2^{*}} d x+o(1) .
\end{aligned}
$$

By changing variables, we obtain

$$
\begin{aligned}
\int_{\mathbb{R}^{N}} \phi_{n}(x)\left|\mathcal{T}_{n} u_{n}\right|^{2^{*}} & =\int_{\mathbb{R}^{N}} \phi(x)\left|u_{n}\right|^{2^{*}} d x \\
& =\int_{\Omega}|x|^{\alpha}\left|u_{n}\right|^{2^{*}} d x \\
\int_{\mathbb{R}^{N}} \phi_{n}(x)|u|^{2^{*}} d x & =\int_{\mathbb{R}^{N}} \phi(q)|u|^{2^{*}} d x+o(1) \\
& =|q|^{\alpha} \int_{\mathbb{R}^{N}}|u|^{2^{*}} d x+o(1) .
\end{aligned}
$$

Inserting these into (118), we get

$$
\begin{aligned}
\int_{\mathbb{R}^{N}} & \phi_{n}(x)\left|\mathscr{T}_{n} u_{n}-\chi_{n} u\right|^{2^{*}} d x \\
= & \int_{\Omega}|x|^{\alpha}\left|u_{n}\right|^{2^{*}} d x-|q|^{\alpha} \int_{\Omega}|u|^{2^{*}} d x+o(1),
\end{aligned}
$$

which, combined with (116), yields (i).

(ii) Without loss of generality, we assume that $u_{n}-$ $\mathscr{T}_{n}^{-1}\left(\chi_{n} u\right)$ is nonnegative; otherwise, one replaces its $\left(2^{*}-1\right)$ th power by $\left|u_{n}-\mathscr{T}_{n}^{-1}\left(\chi_{n} u\right)\right|^{2^{*}-2}\left(u_{n}-\mathscr{T}_{n}^{-1}\left(\chi_{n} u\right)\right)$. Taking $\psi \in$ $C_{0}^{\infty}(\Omega)$,

$$
\begin{aligned}
& \left\langle J_{\alpha}^{\prime}\left(u_{n}-\mathscr{T}_{n}^{-1}\left(\chi_{n} u\right)\right), \psi\right\rangle \\
& =\int_{\mathbb{R}^{N}} \Delta\left(u_{n}-\mathscr{T}_{n}^{-1}\left(\chi_{n} u\right)\right) \Delta \psi d x \\
& \quad-\int_{\mathbb{R}^{N}} \phi(x)\left(u_{n}-\mathscr{T}_{n}^{-1}\left(\chi_{n} u\right)\right)^{2^{*}-1} \psi d x .
\end{aligned}
$$

Since $\chi_{n} u \rightarrow u$ in $D^{2,2}\left(\mathbb{R}^{N}\right)$, we have

$$
\begin{aligned}
& \int_{\mathbb{R}^{N}} \Delta\left(u_{n}-\mathscr{T}_{n}^{-1}\left(\chi_{n} u\right)\right) \Delta \psi d x \\
& =\int_{\mathbb{R}^{N}} \Delta u_{n} \Delta \psi d x-\int_{\mathbb{R}^{N}} \Delta u \Delta\left(\mathscr{T}_{n} \psi\right) d x \\
& \quad+o(1) \int_{\mathbb{R}^{N}}|\Delta \psi|^{2} d x, \\
& \int_{\mathbb{R}^{N}} \phi(x)\left(u_{n}-\mathscr{T}_{n}^{-1}\left(\chi_{n} u\right)\right)^{2^{*}-1} \psi d x \\
& =\int_{\mathbb{R}^{N}} \phi_{n}(x)\left(\mathscr{T}_{n} u_{n}-u\right)^{2^{*}-1} \mathscr{T}_{n} \psi d x \\
& \quad+o(1) \int_{\mathbb{R}^{N}}|\Delta \psi|^{2} d x .
\end{aligned}
$$

By the Brézis-Lieb lemma, we have

$$
\begin{aligned}
\int_{\mathbb{R}^{N}} & \phi_{n}(x)\left(\mathscr{T}_{n} u_{n}-u\right)^{2^{*}-1} \mathscr{T}_{n} \psi d x \\
= & \int_{\mathbb{R}^{N}} \phi_{n}(x)\left(\mathscr{T}_{n} u_{n}\right)^{2^{*}-1} \mathscr{T}^{n} \psi d x \\
& -\int_{\mathbb{R}^{N}} \phi_{n}|u|^{2^{*}-1} \mathscr{T}_{n} \psi d x+o(1) \int_{\mathbb{R}^{N}}|\Delta \psi|^{2} d x
\end{aligned}
$$

By (123), (124), and $\phi_{n} \rightarrow \phi(q)$, we obtain

$$
\begin{aligned}
\int_{\mathbb{R}^{N}} & \phi(x)\left(u_{n}-\mathscr{T}^{-1}\left(\chi_{n} u\right)\right)^{2^{*}-1} \psi d x \\
= & \int_{\mathbb{R}^{N}} \phi(x)\left|u_{n}\right|^{2^{*}-1} \psi d x \\
& \quad-|q|^{\alpha} \int_{\mathbb{R}^{N}}|u|^{2^{*}-1} \mathcal{T}_{n} \psi d x+o(1) \int_{\mathbb{R}^{N}}|\Delta \psi|^{2} d x .
\end{aligned}
$$

Combing (122) and (125), we have

$$
\begin{aligned}
& \left\langle J_{\alpha}^{\prime}\left(u_{n}-\mathscr{T}_{n}^{-1}\left(\chi_{n} u\right)\right), \psi\right\rangle \\
& =\left\langle J_{\alpha}^{\prime}\left(u_{n}\right), \psi\right\rangle-\left\langle J_{\alpha, q}^{\prime}(u), \mathscr{T}_{n} \psi\right\rangle \\
& \quad+o(1) \int_{\mathbb{R}^{N}}|\Delta \psi|^{2} d x \\
& =\left\langle J_{\alpha}^{\prime}\left(u_{n}\right), \psi\right\rangle+o(1) \int_{\mathbb{R}^{N}}|\Delta \psi|^{2} d x,
\end{aligned}
$$

since $u$ is a critical point of $J_{\alpha, q}$ by Lemma 8 . Thus, we prove (ii).

An immediate consequence of (i) and (ii) is the the sequence $\left\{u_{n}-\mathscr{T}_{n}^{-1}\left(\chi_{n} u\right)\right\}$ which is a Palais-Smale sequence for $J_{\alpha}$ at level $(2 / N) \Sigma_{k}^{N / 4}-J_{\alpha, q}(u)$.

We are now ready to describe the behavior of Palais-Smale sequence of $J_{\alpha}$.

Lemma 11. Let $\left\{u_{n}\right\}$ be a Palais-Smale sequence for $J_{\alpha}$ at level $(2 / N) \Sigma^{N / 4}$ and $u_{n} \rightarrow 0$ in $H_{0}^{2}(\Omega)$. Then there is a positive $m$ 
(depending only on $\Sigma_{k}$ ) such that, for every $j=1,2, \ldots, m$, there exist sequences $\left\{\lambda_{j n}\right\} \subset \mathbb{R}^{+}$and $\left\{q_{j n}\right\} \subset \bar{\Omega}$, with $\lambda_{j n} \rightarrow$ $\infty$ and $q_{j n} \rightarrow q_{j} \in \bar{\Omega} \backslash\{0\}$ as $n \rightarrow \infty$, and there exists a nontrivial critical point $v_{j} \in D^{2,2}\left(\mathbb{R}^{N}\right)$ of $J_{\alpha, q_{j}}$ such that (up to subsequence)

$$
\begin{gathered}
u_{n}=\sum_{j=1}^{m} \mathscr{T}_{j n}^{-1}\left(v_{j}\right)+o(1) \quad \text { in } D^{2,2}\left(\mathbb{R}^{N}\right), \\
J_{\alpha}\left(u_{n}\right)=\sum_{j=1}^{m} J_{\alpha, q_{j}}\left(v_{j}\right)+o(1),
\end{gathered}
$$

where $\mathscr{T}_{j n}=\mathscr{T}\left(\lambda_{j n}, q_{j n}\right)$.

Proof. It is clear that there exists a sequence of positive numbers $\lambda_{n} \rightarrow \infty$, a sequence $q_{1 n}$ of points of $\bar{\Omega}$ with $q_{1 n} \rightarrow q_{1} \in \bar{\Omega} \backslash\{0\}$, and a nontrivial critical point $v_{1}$ of $J_{\alpha, q_{1}}$ such that, setting $\mathscr{T}_{1 n}=\mathscr{T}\left(\lambda_{1 n}, q_{1 n}\right)$, the sequence

$$
w_{1 n}:=u_{n}-\mathscr{T}_{1 n}^{-1}\left(\chi_{1 n} v_{1}\right)
$$

is a Palais-Smale sequence for $J_{\alpha}$ at level $(2 / N) \Sigma^{N / 4}-J_{\alpha}\left(v_{1}\right)$.

We now iterate this scheme. If $w_{1 n} \rightarrow 0$ strongly in $L^{2^{*}}\left(\mathbb{R}^{N}\right)$, then the fact that it is a Palais-Smale sequence implies that $w_{1 n} \rightarrow 0$ strongly in $D^{2,2}\left(\mathbb{R}^{N}\right)$. Since also $\chi_{1 n} v_{1} \rightarrow v_{1}$ strongly in $D^{2,2}\left(\mathbb{R}^{N}\right)$, we can write

$$
u_{n}=\mathscr{T}_{1 n}^{-1}\left(v_{1}\right)+o(1) \quad \text { in } D^{2,2}\left(\mathbb{R}^{N}\right),
$$

and the lemma is proved with $m=1$. Otherwise, $w_{1 n} \rightarrow 0$ weakly in $L^{2^{*}}\left(\mathbb{R}^{N}\right)$ but not strongly. In this case, starting with Lemma 10.1 in [17], we can work on $w_{1 n}$ as we did for $u_{n}$. So we can find sequences $\lambda_{2 n} \rightarrow \infty, q_{2 n} \rightarrow q_{2} \in \bar{\Omega} \backslash\{0\}$ and a nontrivial critical point $v_{2}$ of $J_{\alpha, q_{2}}$ such that the sequence

$$
w_{2 n}:=w_{1 n}-\mathscr{T}_{2 n}^{-1}\left(\chi_{2 n} v_{2}\right)
$$

is a Palais-Smale sequence for $J_{\alpha}$ at level $(2 / N) \Sigma_{k}^{N / 4}-$ $J_{\alpha, q_{1}}\left(v_{1}\right)-J_{\alpha, q_{2}}\left(v_{2}\right)$. Once again, if $w_{2 n} \rightarrow 0$ strongly in $L^{2^{*}}\left(\mathbb{R}^{N}\right)$, then we obtain

$$
\begin{array}{r}
u_{n}=\mathscr{T}_{1 n}^{-1}\left(v_{1}\right)+w_{1 n}=\mathscr{T}_{1 n}^{-1}\left(v_{1}\right)+\mathscr{T}_{2 n}^{-1}\left(v_{2}\right)+o(1) \\
\operatorname{in~} D^{2,2}\left(\mathbb{R}^{N}\right),
\end{array}
$$

and the lemma is proved with $m=2$. Otherwise, $w_{2 n} \rightarrow 0$ weakly in $L^{2^{*}}\left(\mathbb{R}^{N}\right)$ but not strongly, and we iterate the above argument. This procedure will end after a finite number of steps. Actually, notice that, by Remark 9 , for all $j$,

$$
\begin{aligned}
J_{\alpha, q_{j}} & =J_{\alpha, q_{j}}\left(\left|q_{j}\right|^{\alpha(4-N) / 8} U\right) \\
& =\left|q_{j}\right|^{\alpha(4-N) / 4} \frac{2}{N} S^{N / 4} \geq \frac{2}{N} S^{N / 4},
\end{aligned}
$$

by definition of $U$, so that, after at most $m:=\left[\left(\Sigma_{k} / S\right)^{N / 4}\right]$ steps, the remainder will be a Palais-Smale sequence at level zero; namely, it will be $o(1)$ in $D^{2,2}\left(\mathbb{R}^{N}\right)$, obtaining the requested representation for $u_{n}$ and $J_{\alpha}\left(u_{n}\right)$.
Remark 12. Checking the process of the proof of Lemma 11, it is easy to see that if one does not suppress the cut-off functions $\chi_{j n}$, one can obtain the following representation of $u_{n}$ :

$$
\begin{array}{r}
u_{n}=\sum_{j=1}^{m} \mathscr{T}_{j n}^{-1}\left(\chi_{j n} v_{j}\right)+o(1) \\
=\sum_{j=1}^{m} \lambda_{j n}^{(N-4) / 2}\left(\chi_{j n} v_{j}\right)\left(\lambda_{j n}\left(\cdot-q_{j n}\right)\right)+o(1) \\
\operatorname{in~} H_{0}^{2}(\Omega) .
\end{array}
$$

Lemma 13. Let $\left\{u_{n}\right\} \subset H_{k}$ be a minimizing sequence for problem (9) and $u_{n} \rightarrow u$ weakly in $H_{0}^{2}(\Omega)$. If $u \neq \equiv$, then $u$ is a minimum point and $u_{n} \rightarrow u$ strongly in $H_{0}^{2}(\Omega)$.

Proof. Notice first that $u \in H_{k}$ since $H_{k}$ is weakly closed in $H_{0}^{2}(\Omega)$. Weak convergence and the Brézis-Lieb Lemma yield

$$
\begin{gathered}
\lim _{n \rightarrow \infty}\left(\int_{\Omega}\left|\Delta u_{n}\right|^{2} d x-\int_{\Omega}\left|\Delta u_{n}-\Delta u\right|^{2} d x\right)=\int_{\Omega}|\Delta u|^{2} d x, \\
\lim _{n \rightarrow \infty}\left(\int_{\Omega}|x|^{\alpha}\left|u_{n}\right|^{2^{*}} d x-\int_{\Omega}|x|^{\alpha}\left|u_{n}-u\right|^{2^{*}} d x\right) \\
=\int_{\Omega}|x|^{\alpha}|u|^{2^{*}} d x .
\end{gathered}
$$

Therefore,

$$
\begin{aligned}
& Q_{\alpha}(u) \\
& \qquad=\frac{\int_{\Omega}\left|\Delta u_{n}\right|^{2} d x-\int_{\Omega}\left|\Delta u_{n}-\Delta u\right|^{2} d x+o(1)}{\left(\int_{\Omega}|x|^{\alpha}\left|u_{n}\right|^{2^{*}} d x-\int_{\Omega}|x|^{\alpha}\left|u_{n}-u\right|^{2^{*}} d x+o(1)\right)^{2 / 2^{*}}} .
\end{aligned}
$$

But $\left\{u_{n}\right\}$ is a minimizing sequence so that

$$
\int_{\Omega}\left|\Delta u_{n}\right|^{2} d x=\Sigma_{k}\left(\int_{\Omega}|x|^{\alpha}\left|u_{n}\right|^{2^{*}} d x\right)^{2 / 2^{*}}+o(1) .
$$

Since $u_{n}-u \in H_{k}$, we have

$$
\int_{\Omega}\left|\Delta\left(u_{n}-u\right)\right|^{2} d x \geq \Sigma_{k}\left(\int_{\Omega}|x|^{\alpha}\left|u_{n}-u\right|^{2^{*}} d x\right)^{2 / 2^{*}}+o(1) .
$$


Inserting (136) and (137) into (135), we obtain

$$
\begin{aligned}
& Q_{\alpha}(u) \\
& \qquad \Sigma_{k}\left(\int_{\Omega}\left|\Delta u_{n}\right|^{2} d x-\int_{\Omega}\left|\Delta u_{n}-\Delta u\right|^{2} d x+o(1)\right) \\
& \quad \times\left(\left[\left(\int_{\Omega}\left|\Delta u_{n}\right|^{2} d x\right)^{2^{*} / 2}\right.\right. \\
& \left.\left.\quad-\left(\int_{\Omega}\left|\Delta\left(u_{n}-u\right)\right|^{2} d x\right)^{2^{*} / 2}+o(1)\right]^{2 / 2^{*}}\right)^{-1} .
\end{aligned}
$$

Since $\int_{\Omega}\left|\Delta u_{n}-\Delta u\right|^{2} d x<\int_{\Omega}\left|\Delta u_{n}\right|^{2} d x$ for $n$ large enough, it is easy to see that $\int_{\Omega}\left|\Delta u_{n}-\Delta u\right|^{2} d x \rightarrow 0$ as $n \rightarrow \infty$. Otherwise, the right hand side of (138) will be strictly less than $\Sigma_{k}$, which contradicts the definition of $\Sigma_{k}$. Thus, we have $u_{n} \rightarrow u$ strongly in $H_{0}^{2}(\Omega)$.

The following proposition is the main result of this section.

Proposition 14. If $\Sigma_{k}<k^{4 / N} S$, then $\Sigma_{k}$ is achieved.

Proof. Let $\left\{u_{n}\right\} \subset H_{k}$ be a (bounded) minimizing sequence for $Q_{\alpha}$ over $H_{k}$. Then there exists a subsequence (still denoted by $\left.u_{n}\right)$ such that $u_{n} \rightarrow u$ in $H_{0}^{2}(\Omega)$. If $u \neq \equiv 0$, by Lemma 13 , $u$ is a minimum point in $H_{k}$, and the proof is completed. If, on the contrary, $u_{n} \rightarrow 0$ in $H_{0}^{2}(\Omega)$, then, as we did above, without loss of generality we can assume that $\left\{u_{n}\right\}$ is a PalaisSmale sequence for $J_{\alpha}$ at level $(2 / N) \Sigma_{k}^{N / 4}$. The behavior of such sequence is described in Lemma 11. In particular,

$$
\begin{aligned}
J_{\alpha}\left(u_{n}\right) & =\sum_{j=1}^{m} J_{\alpha, q_{j}}\left(v_{j}\right)+o(1) \\
& =\sum_{j=1}^{m}\left|q_{j}\right|^{\alpha(4-N) / 4} \frac{2}{N} S^{N / 4}+o(1) \\
& \geq \frac{2 m}{N} S^{N / 4}+o(1) .
\end{aligned}
$$

Since $v_{j}$ is a multiple of the radial function $U$, the cut-off function $\chi_{j n}$ is also radial, and $\mathbf{O}(N-2)$ is a continuous group, we obtain that, for every $j=1, \ldots, m, \lambda_{j n} \operatorname{dist}\left(q_{j n}, \mathbb{R}^{2} \times\right.$ $\{0\}) \rightarrow 0$ as $n \rightarrow 0$. Otherwise, (133) would be incompatible with the symmetry properties of $u_{n}$. Thus, we can replace $q_{j n}$ in (133) with its projection on $\mathbb{R}^{2} \times\{0\}$ so that we can assume that (133) holds in $H_{k}$. This means that $m$ must be a multiple of $k$; say, $m=l k$, for some integer $l \geq 1$. But, from (139),

$$
\begin{aligned}
\frac{2}{N} \Sigma_{k}^{N / 4} & =J_{\alpha}\left(u_{n}\right)+o(1) \\
& \geq l k \frac{2}{N} S^{N / 4}+o(1) \geq \frac{2 k}{N} S^{N / 4}+o(1) ;
\end{aligned}
$$

namely, $\Sigma_{k} \geq k^{4 / N} S$, which is impossible.

\section{Proof of Theorem 1}

This last section is devoted to the proof of Theorem 1 .

Lemma 15. For any $2 \leq p \leq 2^{*}$, there exists $C>0$ depending on $N$ and $p$ such that

$$
\begin{array}{r}
\inf _{u \in H_{0, \text { rad }}^{2}(\Omega) \backslash\{0\}} Q_{\alpha, p}(u) \geq C\left(\frac{\alpha+N}{N}\right)^{1+2 / p} \\
\text { as } \alpha \longrightarrow+\infty,
\end{array}
$$

where $H_{0, \text { rad }}^{2}(\Omega)$ denotes the space of radial functions in $H_{0}^{2}(\Omega)$ and

$$
Q_{\alpha, p}(u)=\frac{\int_{\Omega}|\Delta u|^{2} d x}{\left(\int_{\Omega}|x|^{\alpha}|u|^{p} d x\right)^{2 / p}}
$$

Proof. Let $u \in H_{0, \text { rad }}^{2}(\Omega)$ and define $v(|x|)=u\left(|x|^{\beta}\right)$, where $\beta=N /(\alpha+N)$. Then

$$
\int_{\Omega}|x|^{\alpha}|u|^{p} d x=\beta \int_{\Omega}|v(x)|^{p} d x .
$$

It is easy to see that

$$
\Delta v=\beta\left[\beta u^{\prime \prime}\left(\rho^{\beta}\right) \rho^{2(\beta-1)}+(N+\beta-2) u^{\prime}\left(\rho^{\beta}\right) \rho^{\beta-2}\right] .
$$

We obtain

$$
\begin{aligned}
& \beta^{-1} \int_{\Omega}|\Delta v|^{2}|x|^{(\beta-1)(N-4)} d x \\
& \quad=N \omega_{N} \int_{0}^{1}\left[\beta u^{\prime \prime}(\rho)+(N+\beta-2) \frac{u^{\prime}(\rho)}{\rho}\right]^{2} \rho^{N-1} d \rho .
\end{aligned}
$$

Set

$$
F(\beta)=N \omega_{N} \int_{0}^{1}\left[\beta u^{\prime \prime}(\rho)+(N+\beta-2) \frac{u^{\prime}(\rho)}{\rho}\right]^{2} \rho^{N-1} d \rho .
$$

Direct computations yield $F^{\prime \prime}(\beta)>0$, which shows $F(\beta)$ is convex. Hence

$$
F(\beta) \leq \max \{F(0), F(1)\}
$$

that is

$$
F(\beta) \leq \max \left\{\int_{\Omega} \frac{|\nabla u|^{2}}{|x|^{2}} d x, \int_{\Omega}|\Delta u|^{2} d x\right\} .
$$

By Hardy's inequality, we have

$$
\begin{array}{r}
\int_{\Omega}\left|\nabla \frac{\partial u}{\partial x_{j}}\right|^{2} d x \geq \frac{(N-2)^{2}}{4} \int_{\Omega} \frac{\left|\partial u / \partial x_{j}\right|^{2}}{|x|^{2}} d x \\
j=1,2, \ldots, N
\end{array}
$$


consequently,

$$
\sum_{j=1}^{N} \int_{\Omega}\left|\nabla \frac{\partial u}{\partial x_{j}}\right|^{2} d x \geq \frac{(N-2)^{2}}{4} \int_{\Omega} \frac{|\nabla u|^{2}}{|x|^{2}} d x
$$

Therefore, by (148) and (150), there exists $C>0$ depending only on $N$ such that

$$
\int_{\Omega}|\Delta u|^{2} d x \geq C \beta^{-1} \int_{\Omega}|\Delta v|^{2}|x|^{(\beta-1)(N-4)} d x .
$$

By (143), we have

$$
\begin{gathered}
\frac{\int_{\Omega}|\Delta u|^{2} d x}{\left(\int_{\Omega}|x|^{\alpha}|u|^{p} d x\right)^{2 / p}} \\
\geq C \beta^{-1} \frac{\int_{\Omega}|\Delta v|^{2}|x|^{(\beta-1)(N-4)} d x}{\beta^{2 / p}\left(\int_{\Omega}|v(x)|^{p} d x\right)^{2 / p}} \\
=\frac{C}{\beta^{1+2 / p}} \frac{\int_{\Omega}|\Delta v|^{2}|x|^{(\beta-1)(N-4)} d x}{\left(\int_{\Omega}|v(x)|^{p} d x\right)^{2 / p}} .
\end{gathered}
$$

For every $\beta \in[0,1]$,

$$
c_{\beta}=\inf _{v \in H_{0, \mathrm{rad}}^{2}(\Omega) \backslash\{0\}} \frac{\int_{\Omega}|\Delta v|^{2}|x|^{(\beta-1)(N-4)} d x}{\left(\int_{\Omega}|v(x)|^{p} d x\right)^{2 / p}}
$$

is achieved by standard arguments. Since $c_{\beta}$ is nondecreasing on $[0,1]$, by (152), we obtain (141).

We are now ready for the main result of the paper.

Proof of Theorem 1. For every $\alpha>0$, problem (1) has a solution in some $H_{k}$. Indeed, given $\alpha>0$, there exists $k_{\alpha}>0$ such that, for $k \geq K_{\alpha}$,

$$
\Sigma_{k}<k^{4 / N} S \text {. }
$$

By Proposition $14, \Sigma_{k}$ is achieved by a function $u \in H_{k}$. By invariance, $u$ is a critical point of $Q_{\alpha}$ on $H_{0}^{2}(\Omega)$ which, after scaling, gives rise to a weak solution of (1). By [13], $u$ is a classical solution. We have to show that, at least for $\alpha$ large, $u$ is not radial.

By Lemma 15,

$$
\inf _{u \in H_{0, \mathrm{rad}}^{2}(\Omega) \backslash\{0\}} Q_{\alpha}(u) \geq C \alpha^{(2 N-4) / N},
$$

where the constant $C$ depends only on $N$. We now show that the level $\Sigma_{k}$ of the solution we find is strictly below this threshold for $\alpha$ large. To this aim, we must evaluate how large $k_{\alpha}$ of Proposition 7 has to be in terms of $\alpha$. If we choose $k$ of the order of $\alpha^{4(N-2) / 3(N-5)+1 /(N-5)}$, we see that (102) essentially becomes

$$
\begin{aligned}
R \leq & \frac{3 S^{N / 4}}{\alpha^{3 / 4(N-2)}}-\left(1-\frac{6}{\alpha^{3 / 4(N-2)}}\right) \\
& \cdot \frac{C_{N} a_{0} C_{2}}{2} \alpha^{-(4(N-2) / 3(N-5)+1 /(N-5)) \varepsilon(N-4)} \\
& +O\left(\alpha^{-(4(N-2) / 3(N-5)+1 /(N-5)) \varepsilon(N-2)}\right) .
\end{aligned}
$$

Therefore, $R$ will be negative for all $\alpha$ big enough when $\varepsilon$ is sufficiently small, so we find a solution to (1) in the corresponding $H_{k}$. Therefore, we find a solution at level

$$
\begin{aligned}
\Sigma_{k} & <k^{4 / N} S \leq C \alpha^{4(4 N-3) / 3 N(N-5)} \\
& \leq C \alpha^{(2 N-4) / N} \leq \inf _{u \in H_{0, \mathrm{rad}}^{2}(\Omega) \backslash\{0\}} Q_{\alpha}(u)
\end{aligned}
$$

for all $\alpha$ large enough since $4(4 N-3) / 3 N(N-5) \leq(2 N-4) / N$ for all $N \geq 8$. Therefore, our solution cannot be radial.

\section{Conflict of Interests}

The authors declare that there is no conflict of interests regarding the publication of this paper.

\section{Acknowledgments}

This work is supported by NNSFC (no. 61374089), NSF of Shanxi Province (no. 2014011005-2), and International Cooperation Projects of Shanxi Province (no. 2014081026).

\section{References}

[1] H.-Ch. Grunau, Polyharmonische Dirichletprobleme: Positivität, kritische Exponenten und kritische Dimensionen, Habilitationsschrift, Universität Bayreuth, 1996.

[2] F. Gazzola, H. Grunau, and M. Squassina, "Existence and nonexistence results for critical growth biharmonic elliptic equations," Calculus of Variations and Partial Differential Equations, vol. 18, no. 2, pp. 117-143, 2003.

[3] F. Bernis, J. García Azorero, and I. Peral, "Existence and multiplicity of nontrivial solutions in semilinear critical problems of fourth order," Advances in Differential Equations, vol. 1, no. 2, pp. 219-240, 1996.

[4] T. Bartsch, T. Weth, and M. Willem, "A Sobolev inequality with remainder term and critical equations on domains with topology for the polyharmonic operator," Calculus of Variations and Partial Differential Equations, vol. 18, no. 3, pp. 253-268, 2003.

[5] A. Bahri and J. Coron, "On a nonlinear elliptic equation involving the critical Sobolev exponent: the effect of the topology of the domain," Communications on Pure and Applied Mathematics, vol. 41, no. 3, pp. 253-294, 1988.

[6] E. Berchio, F. Gazzola, and T. Weth, "Radial symmetry of positive solutions to nonlinear polyharmonic Dirichlet problems," Journal für die Reine und Angewandte Mathematik, vol. 620, pp. 165-183, 2008.

[7] W. M. Ni, "A nonlinear Dirichlet problem on the unit ball and its applications," Indiana University Mathematics Journal, vol. 31, no. 6, pp. 801-807, 1982.

[8] J. Byeon and Z. Wang, "On the Hénon equation: asymptotic profile of ground states, I," Annales de l'Institut Henri Poincaré. Analyse Non Linéaire, vol. 23, no. 6, pp. 803-828, 2006.

[9] J. Byeon and Z. Wang, "On the Hénon equation: asymptotic profile of ground states, II," Journal of Differential Equations, vol. 216, no. 1, pp. 78-108, 2005.

[10] D. Cao and S. Peng, "The asymptotic behaviour of the ground state solutions for Hénon equation," Journal of Mathematical Analysis and Applications, vol. 278, no. 1, pp. 1-17, 2003. 
[11] D. Smets, M. Willem, and J. Su, "Non-radial ground states for the Hénon equation," Communications in Contemporary Mathematics, vol. 4, no. 3, pp. 467-480, 2002.

[12] E. Serra, "Non radial positive solutions for the Hénon equation with critical growth," Calculus of Variations and Partial Differential Equations, vol. 23, no. 3, pp. 301-326, 2005.

[13] S. Luckhaus, "Existence and regularity of weak solutions to the Dirichlet problem for semilinear elliptic systems of higher order," Journal für die Reine und Angewandte Mathematik, vol. 306, pp. 192-207, 1979.

[14] S. Agmon, A. Douglis, and L. Nirenberg, "Estimates near the boundary for solution of elliptic partial differential equations satisfying general boundary conditions I," Communications on Pure and Applied Mathematics, vol. 12, pp. 623-727, 1959.

[15] F. Gazzola, H. Grunau, and G. Sweers, Polyharmonic Boundary Value Problems, vol. 1991 of Lecture Notes in Mathematics, Springer, 2010.

[16] H. Grunau and G. Sweers, "Positivity for equations involving polyharmonic operators with Dirichlet boundary conditions," Mathematische Annalen, vol. 307, no. 4, pp. 589-626, 1997.

[17] K. Tintarev and K.-H. Fieseler, Concentration Compactness, Functional-Analytic Grounds and Applications, Imperial College Press, London, UK, 2007.

[18] C. A. Swanson, "The best Sobolev constant," Applicable Analysis, vol. 47, no. 4, pp. 227-239, 1992.

[19] H. Brézis and E. Lieb, "A relation between pointwise convergence of functions and convergence of functionals," Proceedings of the American Mathematical Society, vol. 88, no. 3, pp. 486490, 1983. 


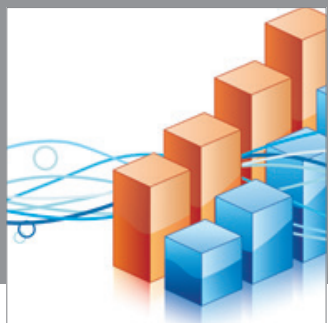

Advances in

Operations Research

mansans

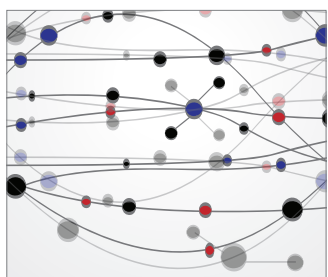

The Scientific World Journal
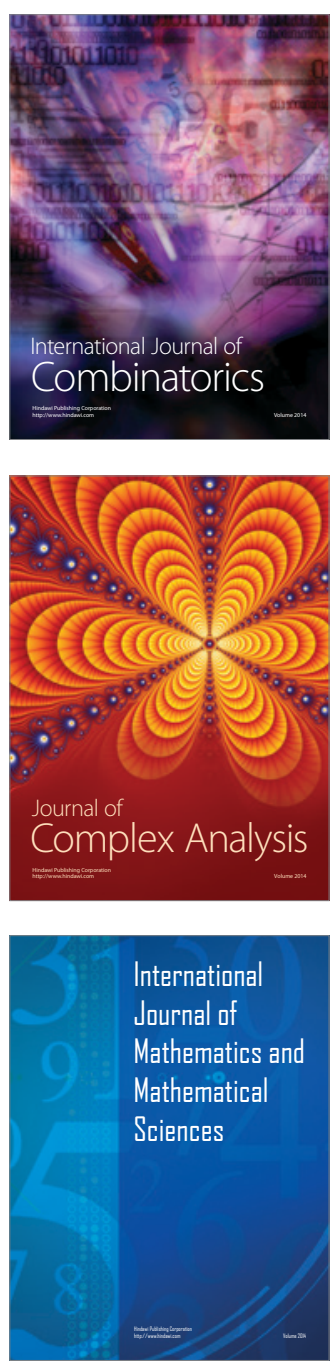
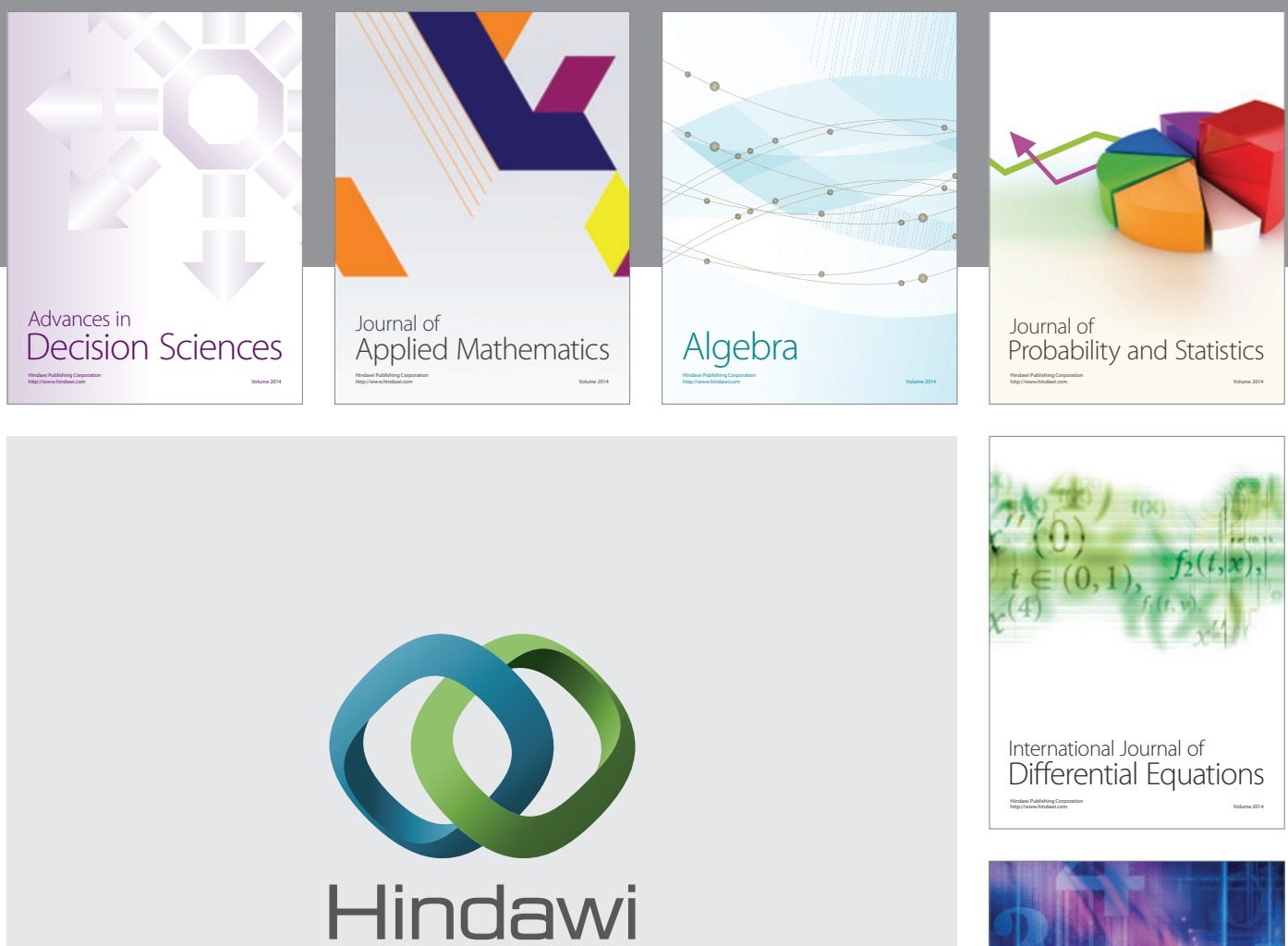

Submit your manuscripts at http://www.hindawi.com
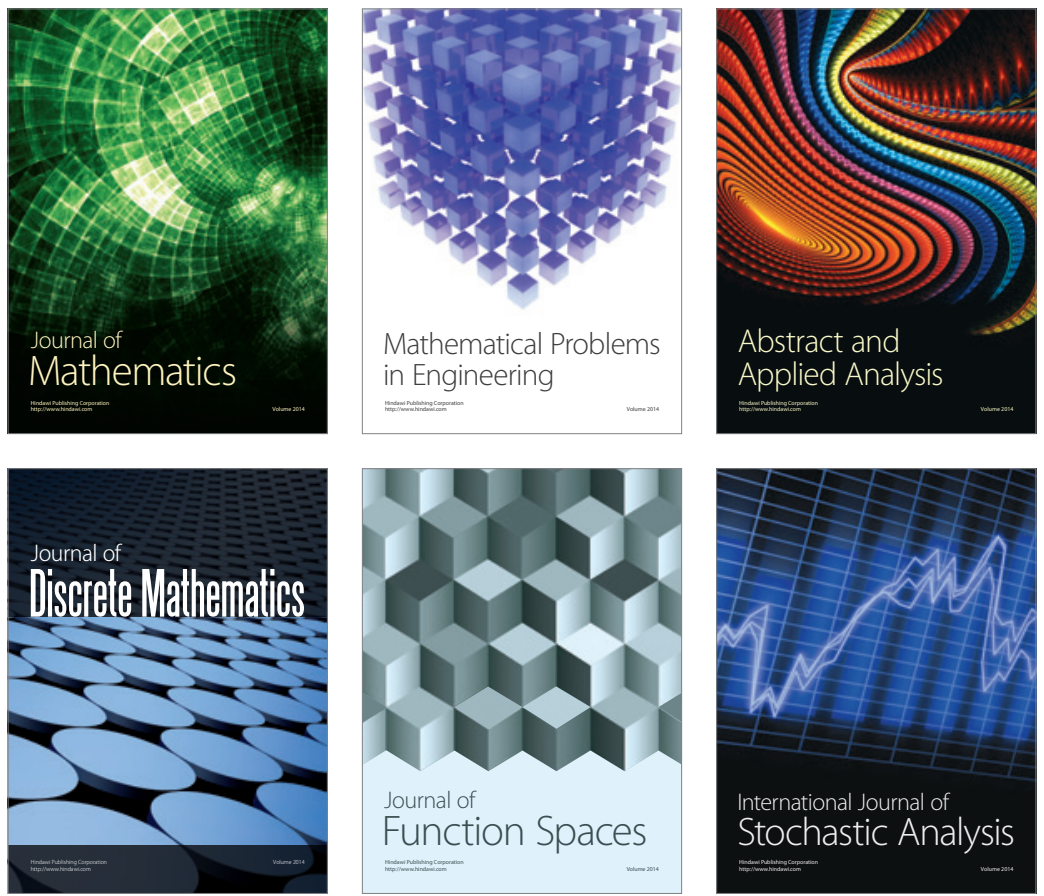

Journal of

Function Spaces

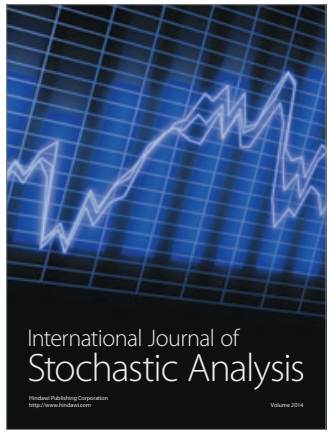

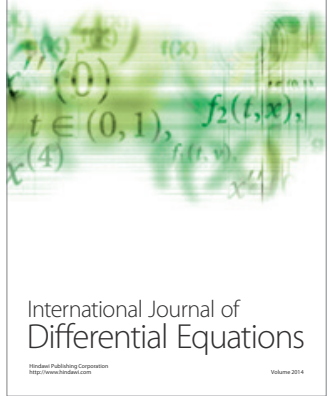
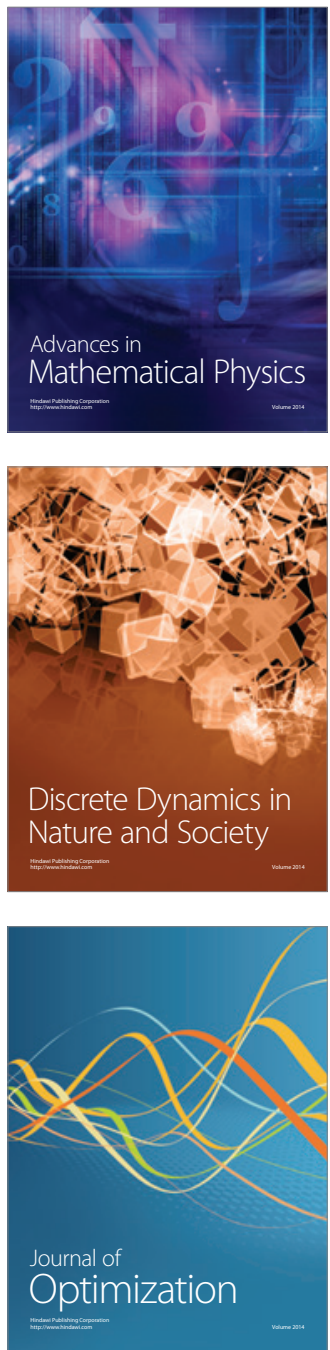